\title{
Tandem Functionalization of Non-activated Alkenes and Alkynes in Intramolecular $N$-Acyloxyiminium Ion Carbocyclization. Synthesis of 6-Substituted Hydroindole 2-Carboxylic acids
}

\author{
Stephen Hanessian, * Martin Tremblay
}

Supplemental Information

1. Experimental procedures (21 pages)

2. X-ray crystal structure data (6 pages)

3. Copies of ${ }^{1} \mathrm{H}$ and ${ }^{13} \mathrm{C}$ NMR spectra (54 pages)

(Spectra of $N$-Boc derivatives consist of two rotamers in most cases) 


\section{General information}

Solvents were distilled under positive pressure of dry argon before use and dried by standard methods; THF and ether, from $\mathrm{Na}$ /benzophenone; and $\mathrm{CH}_{2} \mathrm{Cl}_{2}$, from $\mathrm{CaCl}_{2}$. All commercially available reagents were used without further purification. All reactions were performed under nitrogen atmosphere. NMR $\left({ }^{1} \mathrm{H},{ }^{13} \mathrm{C}\right)$ spectra were recorded on AMX-300, ARX-400, AV-400 and DMX-600 spectrometers. Low- and high-resolution mass spectra were recorded on VG Micromass, AEI-MS 902 or Kratos MS-50 spectrometers using fast atom bombardement (FAB) or electrospray techniques. Optical rotations were recorded on a Perkin-Elmer 241 polarimeter in a $1 \mathrm{dm}$ cell at ambient temperature. Analytical thin-layer chromatography was performed on Merck $60 \mathrm{~F}_{254}$ pre-coated silica gel plates. Visualization was performed by ultraviolet light and/or by staining with ceric ammonium molybdate, ninhydrine or potassium permanganate. Flash column chromatography was performed using $(40-60 \mu \mathrm{m})$ silica gel at increased pressure.
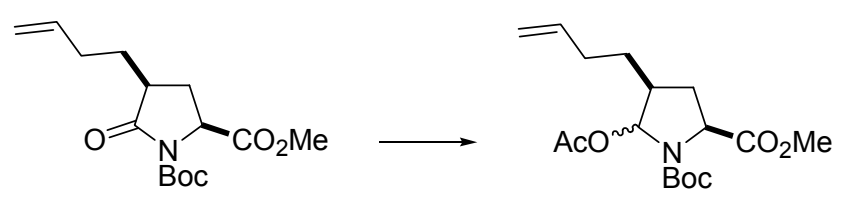

6

(2S,4S)-5-Acetoxy-4-but-3-enyl-pyrrolidine-1,2-dicarboxylic acid 1-tert-butyl ester

2-methyl ester (2). To a solution of the N-Boc lactam $(3.00 \mathrm{~g}, 10.0 \mathrm{mmol})$ in THF (70 $\mathrm{mL}$ ) at $-78^{\circ} \mathrm{C}$, was added a solution of $\mathrm{LiHBEt}_{3}(11.1 \mathrm{~mL}, 1.0 \mathrm{~N}$ in THF), stirred of $1 \mathrm{~h}$, quenched with $\mathrm{NaHCO}_{3}$ (sat.), 2 drops of $\mathrm{H}_{2} \mathrm{O}_{2}\left(30 \%\right.$ in $\left.\mathrm{H}_{2} \mathrm{O}\right)$ were added and the solution was concentrated under vacuum. The aqueous layer was extracted with $\mathrm{CH}_{2} \mathrm{Cl}_{2}$, the combined organic extracts were washed with brine, dried over $\mathrm{Na}_{2} \mathrm{SO}_{4}$ and concentrated under vacuum. The resulting oil was dissolved in $\mathrm{CH}_{2} \mathrm{Cl}_{2}(25 \mathrm{~mL})$ and $\mathrm{Et}_{3} \mathrm{~N}$ (2.81 mL, $20.2 \mathrm{mmol}), \mathrm{Ac}_{2} \mathrm{O}(2.86 \mathrm{~mL}, 30.3 \mathrm{mmol})$ and DMAP (cat.) were added successively. After stirring overnight, the solution was quenched with $\mathrm{NaHCO}_{3}$ (sat.), 
the aqueous layer was extracted with $\mathrm{CH}_{2} \mathrm{Cl}_{2}$, the combined organic extracts were washed with brine, dried over $\mathrm{Na}_{2} \mathrm{SO}_{4}$ and concentrated under vacuum. The crude oil obtained was purified by flash chromatography (EtOAc/Hexanes 15:85) to give the corresponding hemiaminal derivative $2(3.13 \mathrm{~g}, 91 \%)$ as a mixture of diastereoisomeres.

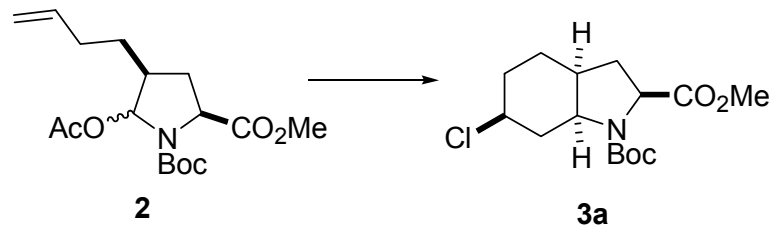

(2S,3aS,6S,7aS)-6-Chloro-2,3,3a,4,5,6,7,7a-octahydroindole-1,2-dicarboxylic acid 1-tert-butyl ester 2-methyl ester ( $\underline{3 a})$. To a solution of the hemiaminal 2 derivative $(0.400 \mathrm{~g}, 1.17 \mathrm{mmol})$ in $\mathrm{CH}_{2} \mathrm{Cl}_{2}(8 \mathrm{~mL})$ at $-78^{\circ} \mathrm{C}$, was added a solution of $\mathrm{SnCl}_{4}(1.52$ Deleted: 7 $\mathrm{mL}, 1.0 \mathrm{M}$ in $\mathrm{CH}_{2} \mathrm{Cl}_{2}$ ) dropwise. After stirring for $10 \mathrm{~min}$., the solution was quenched with $\mathrm{NaHCO}_{3}$ (sat.), warmed up to RT, filtered on a small celite pad, the filtrated was washed with brine, dried over $\mathrm{Na}_{2} \mathrm{SO}_{4}$ and concentrated under vacuum. The crude oil obtained was purified by flash chromatography (EtOAc/Hexanes 10:90 to 15:85) to give 3 a $(0.245 \mathrm{~g}, 66 \%)$ as a colorless oil; ${ }^{1} \mathrm{H}$ RMN (400 MHz, DMSO- $\left.\mathrm{d}_{6}\right) \delta$ (rotamers) 4.20-4.15 (m, 1H), 4.05-3.92 (m, 1H), 3.85-3.75 (m, 1H), $3.64(\mathrm{~s}, 1.5 \mathrm{H}), 3.61(\mathrm{~s}, 1.5 \mathrm{H}), 2.42-2.20$ (m, 2H), 2.16-2.05 (m, 1H), 1.95-1.80 (m, 2H), 1.75-1.60 (m, 4H), $1.17(\mathrm{~s}, 4.5 \mathrm{H}), 1.13$ $(\mathrm{s}, 4.5 \mathrm{H}) ;{ }^{13} \mathrm{C}$ NMR $\left(100 \mathrm{MHz}, \mathrm{DMSO}-\mathrm{d}_{6}\right) \delta$ (rotamers) $174.4,173.9,153.8,153.1,80.1$, $59.9,58.0,57.9,57.8,57.4,52.9,52.8,39.3,39.2,38.9,35.9,35.2,32.5,32.0,31.7$, 29.1, 28.9, 24.8; $[\alpha]_{\mathrm{D}}+2.0^{\circ}$ (c $\left.0.98, \mathrm{CHCl}_{3}\right)$; HRMS for $\mathrm{C}_{15} \mathrm{H}_{25} \mathrm{NO}_{4} \mathrm{Cl}$ calculated $\left(\mathrm{M}+\mathrm{H}^{+}\right)$ 318.14860 found 318.14722 .

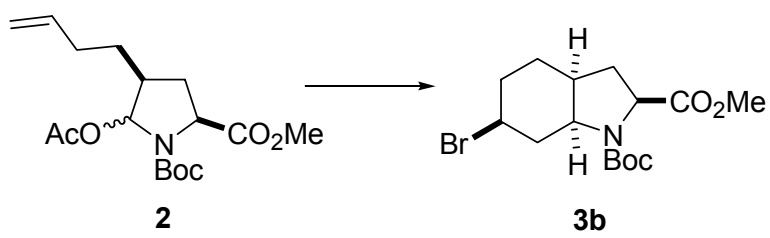


(2S,3aS,6S,7aS)-6-Bromo-2,3,3a,4,5,6,7,7a-octahydroindole-1,2-dicarboxylic acid 1-tert-butyl ester 2-methyl ester (3b). Same procedure as 3a. $(78 \%) ;{ }^{1} \mathrm{H}$ RMN (400 $\left.\mathrm{MHz}, \mathrm{DMSO}-\mathrm{d}_{6}\right) \delta$ (rotamers) 4.21-4.08 $(\mathrm{m}, 2 \mathrm{H}), 3.85-3.79(\mathrm{~m}, 1 \mathrm{H}), 3.67(\mathrm{~s}, 1.5 \mathrm{H}), 3.64$ (s, 1.5H), 2.62-2.41 (m, 1H), 2.39-2.21 (m, 1H), 2.17-1.60 (m, 7H), $1.37(\mathrm{~s}, 4.5 \mathrm{H}), 1.30$ $(\mathrm{s}, 4.5 \mathrm{H}) ;{ }^{13} \mathrm{C}$ NMR $\left(100 \mathrm{MHz}, \mathrm{DMSO}-\mathrm{d}_{6}\right) \delta$ (rotamers) $174.3,173.8,153.6,152.9,80.0$, 79.9, 59.7, 59.3, 58.0, 57.6, 52.8, 52.7, 50.1, 50.0, 39.6, 35.6, 35.0, 32.7, 32.6, 32.3, 31.5, 29.0, 28.7, 25.6; $[\alpha]_{\mathrm{D}}+5.9^{\circ}$ (c 1.5, $\mathrm{CHCl}_{3}$ ); HRMS for $\mathrm{C}_{15} \mathrm{H}_{24} \mathrm{NO}_{4} \mathrm{Br}$ calculated 361.088870 found 361.088652 .

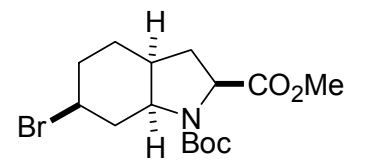

$3 \mathbf{b}$

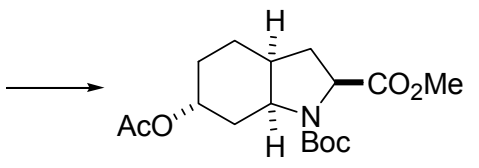

$4 a$
(2S,3aS,6R,7aS)-6-Acetoxy-2,3,3a,4,5,6,7,7a-octahydroindole-1,2-dicarboxylic acid 1-tert-butyl ester 2-methyl ester (4a). To a solution of $3 \mathrm{~b}(0.500 \mathrm{~g}, 1.38 \mathrm{mmol})$ in toluene $(12 \mathrm{~mL})$, was added $\mathrm{Bu}_{4} \mathrm{NOAc}(6.20 \mathrm{~g}, 20.7 \mathrm{mmol})$ and the mixture was heated at $40-50^{\circ} \mathrm{C}$ for $2 \mathrm{~h}$. After cooling down to RT, the solution was diluted with Hexanes, the organic solution was washed with $\mathrm{H}_{2} \mathrm{O}$, brine and dried over $\mathrm{Na}_{2} \mathrm{SO}_{4}$. The solvent was removed under vacuum and the resulting oil was purified by flash chromatography (EtOAc/Hexanes $15: 85)$ to give $4 \mathrm{a}(0.368 \mathrm{~g}, 78 \%)$ as a colorless oil; ${ }^{1} \mathrm{H}$ RMN $(400 \mathrm{MHz}$,
Deleted: $7 b$ Deleted: $7 a$
Deleted: 8a Deleted: $7 \mathrm{~b}$ DMSO-d $\left._{6}\right) \delta$ 4.97-4.90 (m, 1H), 4.22-4.12 (m, 1H), 3.95-3.82 (m, 1H), $3.67(\mathrm{~s}, 3 \mathrm{H}), 2.40-$ $2.28(\mathrm{~m}, 1 \mathrm{H}), 2.20-2.10(\mathrm{~m}, 2 \mathrm{H}), 2.05(\mathrm{~s}, 3 \mathrm{H}), 1.96-1.80(\mathrm{~m}, 2 \mathrm{H}), 1.66-1.47(\mathrm{~m}, 4 \mathrm{H})$, 1.30 (s, 9H); ${ }^{13} \mathrm{C}$ NMR $\left(100 \mathrm{MHz}, \mathrm{DMSO}_{-} \mathrm{d}_{6}\right) \delta 174.3,170.6,79.7,69.8,59.7,59.2,53.9$, 52.7, 36.3, 35.8, 32.5, 31.9, 30.9, 28.7, 24.0, 21.8, 20.4; [ $]_{\mathrm{D}}-47.5^{\circ}$ (c 1.55, $\mathrm{CHCl}_{3}$ ); HRMS for $\mathrm{C}_{17} \mathrm{H}_{28} \mathrm{NO}_{6}$ calculated $\left(\mathrm{M}+\mathrm{H}^{+}\right) 342.191663$ found 342.193100 .<smiles>CC(=O)C1CC2CC[C@@H](N)CC2C1[18O]</smiles> 


\section{(2S,3aS,6R,7aS)-6-Azido-N-tert-butyloxycarbonyl-octahydroindole-2-carboxylate}

de méthyle (4b). To a solution of the bromide $3 \mathrm{~b}(0.051 \mathrm{~g}, 0.141 \mathrm{mmol})$ in DMF $(1 \mathrm{~mL})$, was added $\mathrm{NaN}_{3}(0.183 \mathrm{~g}, 2.82 \mathrm{mmol})$ and the solution was heated at $100^{\circ} \mathrm{C}$ for $24 \mathrm{~h}$. After cooled down to RT, the solution was diluted with EtOAc, the organic layer was washed with $\mathrm{H}_{2} \mathrm{O}(3 \mathrm{X})$, brine and dried over $\mathrm{Na}_{2} \mathrm{SO}_{4}$. The solvent was removed under vacuum and the resulting oil was purified by flash chromatography (EtOAc/Hexanes $15: 85)$ to give the azido $4 \mathrm{~b}(0.032 \mathrm{~g}, 71 \%)$ as a colorless oil; ${ }^{1} \mathrm{H}$ NMR $(400 \mathrm{MHz}$, DMSO- $\left._{6}\right) \delta$ 4.20-4.10 (m, 1H), 4.08-4.01 (m, 1H), 3.63-3.57 (broad s, 3H), 2.37-2.20 (m, 1H), 2.12-2.04 (m, 2H), 1.90-1.72 (m, 2H), 1.70-1.40 (m, 4H), 1.38-1.20 (m, 10H); ${ }^{13} \mathrm{C}$ NMR $(75 \mathrm{MHz}$, DMSO-d 6 ) $\delta 174.3,173.8,153.8,153.1,80.0,59.6,59.1,57.5,57.1$, 54.1, 53.7, 52.7, 36.3, 35.7, 30.5, 28.8, 24.0, 20.6; IR cm ${ }^{-1}$ 2936, 2104, 1751, 1699,

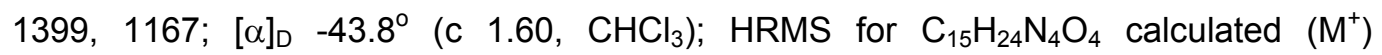
324.179756, found 324.180101.

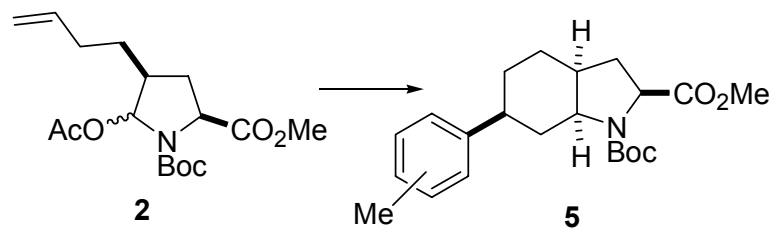

(2S,3aS,6S,7aS)-6-(o/p-Tolyl)-octahydro-indole-1,2-dicarboxylic acid 1-tert-butyl ester 2-methyl ester (5). To a solution of the hemiaminal derivative 2 ( $0.095 \mathrm{~g}, 0.278$ $\mathrm{mmol})$ in toluene $(3 \mathrm{~mL})$ at $-78^{\circ} \mathrm{C}$, was added $\mathrm{BF}_{3} \mathrm{OEt}_{2}(52 \mu \mathrm{L}, 0.417 \mathrm{mmol})$ dropwise. After stirring for 10 min., the solution was quenched with $\mathrm{NaHCO}_{3}$ (sat.), warmed up to $\mathrm{RT}$, diluted with $\mathrm{CH}_{2} \mathrm{Cl}_{2}$, washed with brine, dried over $\mathrm{Na}_{2} \mathrm{SO}_{4}$ and concentrated under vacuum. The crude oil obtained was purified by flash chromatography (EtOAc/Hexanes $20: 80)$ to give $5(0.079 \mathrm{~g}, 77 \%)$ as a colorless oil; ${ }^{1} \mathrm{H}$ NMR $\left(400 \mathrm{MHz}, \mathrm{CDCl}_{3}\right) \delta($ mixture of regioisomers and rotamers) 7.28-6.93 $(\mathrm{m}, 4 \mathrm{H}), 4.34-4.18(\mathrm{~m}, 1 \mathrm{H}), 4.09-3.88(\mathrm{~m}, 1 \mathrm{H})$, 3.78-3.68 (m, 3H), 2.70-2.60 (m, 0.5H), 2.50-2.23 (m, 4.5H), 2.22-1.98 (m, 2H), 1.95$1.78(\mathrm{~m}, 2 \mathrm{H}), 1.77-1.48(\mathrm{~m}, 3 \mathrm{H}), 1.42(\mathrm{~s}, 4.5 \mathrm{H}), 1.37(\mathrm{~s}, 4.5 \mathrm{H}), 1.30-1.20(\mathrm{~m}, 1 \mathrm{H}) ;{ }^{13} \mathrm{C}$ NMR $\left(100 \mathrm{MHz}, \mathrm{CDCl}_{3}\right) \delta$ (mixture of regioisomers and rotamers) 174.4, 174.3, 153.6,
Deleted: 8b

Deleted: $7 a$

Deleted: $8 b$

Deleted: 6

Deleted: 9 
$144.6,138.2,135.8,135.5,130.7,129.5,129.4,128.6,128.1,127.1,126.7,126.5$, $126.3,125.7,124.2,80.2,59.8,59.3,59.2,58.6,58.4,58.2,58.0,52.6,52.4,37.6,36.9$, $36.3,36.2,35.0,34.8,33.0,32.2,28.7,28.5,27.8,26.5,21.4$; HRMS for $\mathrm{C}_{22} \mathrm{H}_{32} \mathrm{NO}_{4}$ calculated $\left(\mathrm{M}+\mathrm{H}^{+}\right) 374.233134$, found 374.232794 .

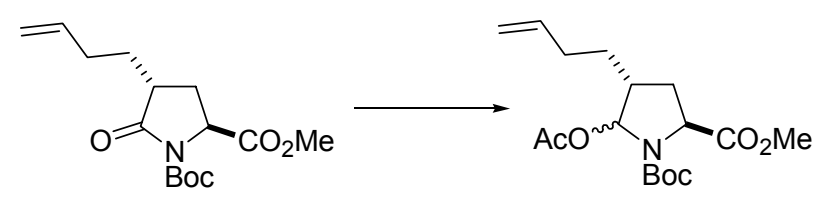

(2S,4R)-5-Acetoxy-4-but-3-enyl-pyrrolidine-1,2-dicarboxylic acid 1-tert-butyl ester 2-methyl ester. Same procedure as for 2 (80\%).

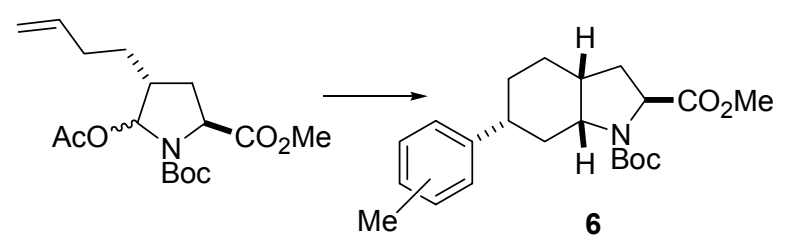

(2S,3aR,6R,7aR)-6-(o/p-Tolyl)-octahydro-indole-1,2-dicarboxylic acid 1-tert-butyl ester 2-methyl ester (ㄸ). Same procedure as for $\underline{5}(74 \%) ;{ }^{1} \mathrm{H}$ NMR $\left(400 \mathrm{MHz}, \mathrm{CDCl}_{3}\right) \delta$ Deleted: 12 (mixture of regioisomers and rotamers) 7.20-6.90 (m, 4H), 4.38-4.30 (m, 0.5H), 4.29Deleted: 9 $4.22(\mathrm{~m}, 0.5 \mathrm{H}), 4.17-3.93(\mathrm{~m}, 1 \mathrm{H}), 3.76-3.70(\mathrm{~m}, 3 \mathrm{H}), 2.68-2.50(\mathrm{~m}, 1 \mathrm{H}), 2.48-2.09(\mathrm{~m}$, $5 \mathrm{H}$ ), $1.90-1.77(\mathrm{~m}, 3 \mathrm{H}), 1.75-1.28(\mathrm{~m}, 13 \mathrm{H}) ;{ }^{13} \mathrm{C} \mathrm{NMR}\left(100 \mathrm{MHz}, \mathrm{CDCl}_{3}\right) \delta$ (mixture of regioisomers and rotamers) 174.2, 173.8, 154.4, 153.5, 144.6, 143.8, 143.7, 135.6, $130.8,130.7,129.6,129.4,128.6,127.9,127.4,127.2,127.0,126.7,126.4,126.3$, $126.2,124.2,124.1,80.2,80.1,60.8,58.7,58.3,58.3,58.0,57.8,57.6,52.6,52.4,37.3$, $36.4,35.2,35.1,34.3,34.2,32.4,31.5,31.4,28.8,28.7,26.3,26.0,21.4$; HRMS for $\mathrm{C}_{22} \mathrm{H}_{32} \mathrm{NO}_{4}$ calculated $\left(\mathrm{M}+\mathrm{H}^{+}\right) 374.233134$, found 374.232887 . 


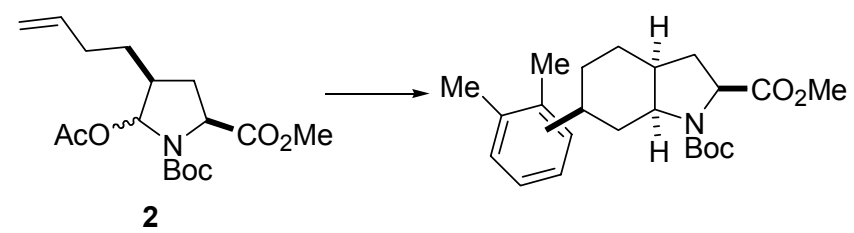

(2S,3aS,6S,7aS)-6-(o/p-1,2-Dimethyl-phenyl)-2,3,3a,4,5,7a-hexahydro-indole-1,2dicarboxylic acid 1-tert-butyl ester 2-methyl ester (Entry 1, table 1). Same procedure as for $\underline{5}$, except that the reaction was done in o-xylene at $-20^{\circ} \mathrm{C}(67 \%) ;{ }^{1} \mathrm{H}$ NMR $(400$ Deleted: 9 $\left.\mathrm{MHz}, \mathrm{CDCl}_{3}\right) \delta$ (mixture of regioisomers and rotamers) 7.12-6.89 $(\mathrm{m}, 3 \mathrm{H}), 4.32-4.18(\mathrm{~m}$, $1 \mathrm{H}), 4.10-3.88(\mathrm{~m}, 1 \mathrm{H}), 3.80-3.69(\mathrm{~m}, 3 \mathrm{H}), 2.80-2.68(\mathrm{~m}, 0.4 \mathrm{H}), 2.50-1.95(\mathrm{~m}, 10.6 \mathrm{H})$, 1.93-1.80 (m, 2H), 1.75-1.25 (m, $\left.12 \mathrm{H}) ;{ }^{13} \mathrm{C} \mathrm{NMR} \mathrm{(100} \mathrm{MHz,} \mathrm{CDCl}_{3}\right) \delta$ (mixture of regioisomers and rotamers) $173.8,173.7,173.6,153.7,152.9,143.8,143.6,136.6$, 136.4 , 134.2, 133.6, 129.5, 129.4, 128.0, 127.6, 127.4, 125.5, 125.3, 124.0, 123.9, 122.9, 105.0, 79.6, 59.3, 58.8, 58.1, 57.8, 57.6, 52.0, 51.8, 41.3, 40.9, 37.4, 36.4, 35.7, $35.3,34.8,32.5,31.7,31.6,28.3,28.1,27.6,26.2,26.0,20.9,19.7,19.2$, ; HRMS for $\mathrm{C}_{23} \mathrm{H}_{34} \mathrm{NO}_{4}$ calculated $\left(\mathrm{M}+\mathrm{H}^{+}\right) 388.248784$, found 388.247856 .

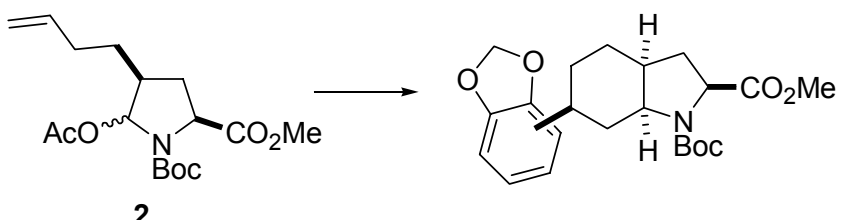

(2S,3aS,6S,7aS)-6-(o/p-Benzo[1,3]dioxolyl)-2,3,3a,4,5,7a-hexahydro-indole-1,2dicarboxylic acid 1-tert-butyl ester 2-methyl ester (Entry 2, table 1). Same procedure as for $\underline{5}$, except that the reaction was done in phthalane at $-15^{\circ} \mathrm{C}(58 \%) ;{ }^{1} \mathrm{H}$ NMR $(400$ $\left.\mathrm{MHz}, \mathrm{CDCl}_{3}\right) \delta$ (mixture of regioisomers and rotamers) 6.80-6.60 (m, 3H), 5.95-5.88 (m, $2 \mathrm{H}), 4.30-4.18(\mathrm{~m}, 1 \mathrm{H}), 4.07-3.97(\mathrm{~m}, 0.6 \mathrm{H}), 3.96-3.87(\mathrm{~m}, 0.4 \mathrm{H}), 3.74-3.67(\mathrm{~m}, 3 \mathrm{H})$, 2.68-2.58 (m, 0.4H), 2.42-2.29 (m, 2.6H), 2.20-2.05 (m, 3H), 1.88-1.50 (m, 4H), 1.48$1.30(\mathrm{~m}, 9 \mathrm{H}) ;{ }^{13} \mathrm{C}$ NMR $\left(100 \mathrm{MHz}, \mathrm{CDCl}_{3}\right) \delta$ (mixture of regioisomers and rotamers) $174.4,154.3,153.5,147.9,140.9,140.8,128.2$, 121.9, 120.2, 119.9, 108.6, 108.5, $107.8,106.8,101.1,100.8,80.2,60.8,59.8,59.3,57.8,52.4,52.3,41.9,41.7,36.4$, 
36.2, 36.1, 35.7, 32.9, 28.8, 28.7, 26.6, 26.2, 14.6; HRMS for $\mathrm{C}_{22} \mathrm{H}_{29} \mathrm{NO}_{6}$ calculated $\left(\mathrm{M}^{+}\right)$ 403.199488, found 403.201268 .

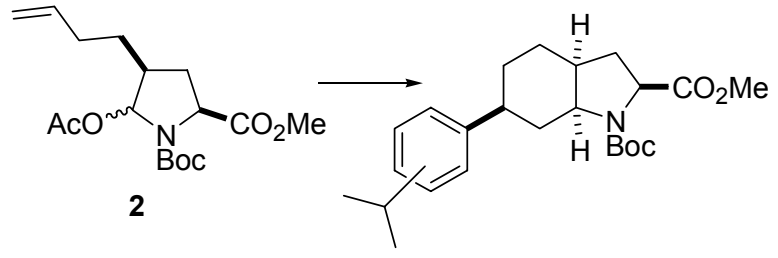

(2S,3aS,6S,7aS)-6-(o/p-lsopropyl-phenyl)-2,3,3a,4,5,7a-hexahydro-indole-1,2dicarboxylic acid 1-tert-butyl ester 2-methyl ester (Entry 3, table 1). Same procedure as for $\underline{5}$, except that the reaction was done in cumene at $-78^{\circ} \mathrm{C}(70 \%) ;{ }^{1} \mathrm{H}$ NMR $(400$ $\left.\mathrm{MHz}, \mathrm{CDCl}_{3}\right) \delta$ (mixture of regioisomers and rotamers) 7.28-7.00 (m, 4H), 4.37-4.18 (m, $1 \mathrm{H}), 4.08-3.88(\mathrm{~m}, 1 \mathrm{H}), 3.79-3.68(\mathrm{~m}, 3 \mathrm{H}), 2.93-2.82(\mathrm{~m}, 1 \mathrm{H}), 2.50-2.30(\mathrm{~m}, 2 \mathrm{H}), 2.25-$ $2.00(\mathrm{~m}, 2 \mathrm{H}), 1.90-1.78(\mathrm{~m}, 2 \mathrm{H}), 1.77-1.34(\mathrm{~m}, 13 \mathrm{H}), 1.30-1.18(\mathrm{~m}, 6 \mathrm{H}) ;{ }^{13} \mathrm{C}$ NMR $(100$ $\left.\mathrm{MHz}, \mathrm{CDCl}_{3}\right) \delta$ (mixture of regioisomers and rotamers) 174.4, 174.2, 154.4, 153.6, $153.5,149.2$, 147.0, 146.6, 144.1, 144.0, 128.8, 128.7, 127.1, 127.0, 126.8, 125.8, $125.7,124.5,124.4,80.2,59.3,58.4,58.2,58.1,52.6,52.4,41.5,36.9,36.2,35.9,35.4$, 34.6, 34.0, 32.9, 32.1, 29.3, 28.9, 28.8, 28.2, 26.5, 26.3, 24.5; HRMS for $\mathrm{C}_{24} \mathrm{H}_{36} \mathrm{NO}_{4}$ calculated $\left(\mathrm{M}+\mathrm{H}^{+}\right)$402.264434, found 402.263991.

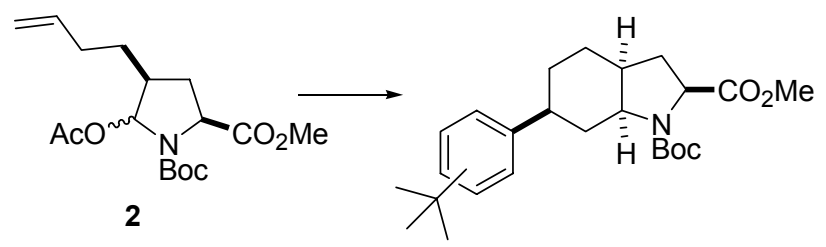

\section{(2S,3aS,6S,7aS)-6-(m/p-tert-Butyl-phenyl)-2,3,3a,4,5,7a-hexahydro-indole-1,2-}

dicarboxylic acid 1-tert-butyl ester 2-methyl ester (Entry 4, table 1). Same procedure as for 5 , except that the reaction was done in tert-butylbenzene at $-78^{\circ} \mathrm{C}(50 \%) ;{ }^{1} \mathrm{H}$ NMR (400 MHz, $\left.\mathrm{CDCl}_{3}\right) \delta$ (mixture of regioisomers and rotamers) 7.37-7.00 (m, 4H), 4.36-4.20 (m, 1H), 4.10-4.00 (m, 0.5H), 3.98-3.89 (m, 0.5H), $3.77(\mathrm{~s}, 1.5 \mathrm{H}), 3.74(\mathrm{~s}$, 
$1.5 \mathrm{H}), 2.55-2.35(\mathrm{~m}, 2.5 \mathrm{H}), 2.34-2.00(\mathrm{~m}, 2.5 \mathrm{H}), 1.92-1.80(\mathrm{~m}, 2 \mathrm{H}), 1.78-1.65(\mathrm{~m}, 1.5 \mathrm{H})$, 1.62-1.20 (m, 19.5H); ${ }^{13} \mathrm{C} \mathrm{NMR}\left(100 \mathrm{MHz}, \mathrm{CDCl}_{3}\right) \delta$ (mixture of regioisomers and rotamers) $174.4,174.2,154.4,153.6,153.5,151.4,149.3,149.1,146.3,143.7,143.6$, $128.5,128.4,126.9,126.8,125.7,125.5,124.6,124.5,123.9,123.8,123.6,123.4,80.2$, $59.9,59.3,58.4,58.2,58.1,52.5,52.3,41.6,41.3,36.9,36.2,36.1,35.9,35.4,34.7$, $32.9,32.1,31.8,29.2,28.9,28.7,28.1,28.0,26.5,26.3$; HRMS for $\mathrm{C}_{25} \mathrm{H}_{37} \mathrm{NO}_{4}$ calculated $\left(\mathrm{M}^{+}\right)$415.272259, found 415.272741 .

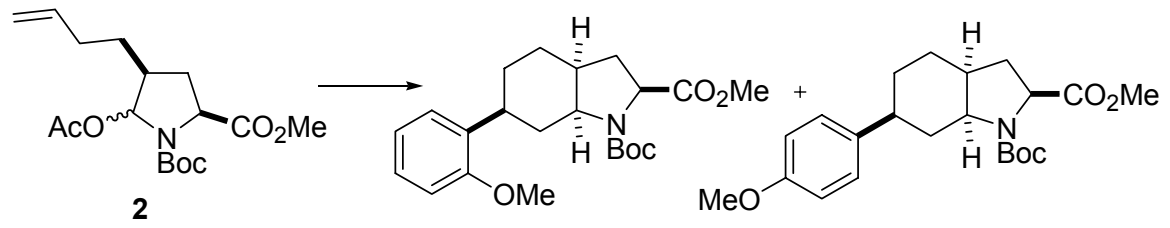

Same procedure as for $\underline{5}$, except that the reaction was done in anisole at $-35^{\circ} \mathrm{C}(69 \%$,

1:1 o/p). (2S,3aS,6S,7aS)-6-(o-Methoxy-phenyl)-octahydro-indole-1,2-dicarboxylic acid 1-tert-butyl ester 2-methyl ester (Entry 5, table 1); ${ }^{1} \mathrm{H} \mathrm{NMR}\left(300 \mathrm{MHz}, \mathrm{CDCl}_{3}\right) \delta$ (rotamers) 7.17-7.05 (m, 2H), 6.83-6.78 (m, 2H), $4.30(\mathrm{t}, 0.5 \mathrm{H}, \mathrm{J}=8.6 \mathrm{~Hz}), 4.22(\mathrm{t}, 0.5 \mathrm{H}$, $J=8.6 \mathrm{~Hz}), 4.08-3.95(\mathrm{~m}, 0.5 \mathrm{H}), 3.95-3.85(\mathrm{~m}, 0.5 \mathrm{H}), 3.82-3.66(\mathrm{~m}, 6 \mathrm{H}), 2.48-2.26(\mathrm{~m}$, $2 \mathrm{H}), 2.22-2.00(\mathrm{~m}, 2 \mathrm{H}), 1.90-1.75(\mathrm{~m}, 2 \mathrm{H}), 1.70-1.30(\mathrm{~m}, 13 \mathrm{H}) ;{ }^{13} \mathrm{C}$ NMR $(75 \mathrm{MHz}$, $\left.\mathrm{CDCl}_{3}\right) \delta$ (rotamers) $174.5,174.4,158.3,158.2,154.4,153.6,139.0,128.1,114.2$, $114.1,80.2,59.8,58.4,58.0,55.8,55.7,52.4,41.3,41.0,36.2,35.9,35.5,32.9,32.1$, 29.4, 28.9, 28.7, 28.6, 26.5, 26.3; $[\alpha]_{\mathrm{D}}-21.9^{\circ}$ (c 1.05, $\mathrm{CHCl}_{3}$ ); HRMS for $\mathrm{C}_{22} \mathrm{H}_{31} \mathrm{NO}_{5}$ calculated $\left(\mathrm{H}^{+}\right)$389.220223, found 389.221982. (2S,3aS,6S,7aS)-6-(p-Methoxyphenyl)-octahydro-indole-1,2-dicarboxylic acid 1-tert-butyl ester 2-methyl ester (Entry 5, table 1); ${ }^{1} \mathrm{H}$ NMR $\left(300 \mathrm{MHz}, \mathrm{CDCl}_{3}\right) \delta$ (rotamers) 7.21-7.05 (m, 2H), 6.94-6.70 $(\mathrm{m}, 2 \mathrm{H}), 4.33-4.17(\mathrm{~m}, 1 \mathrm{H}), 4.15-3.90(\mathrm{~m}, 1 \mathrm{H}), 3.82-3.62(\mathrm{~m}, 6 \mathrm{H}), 2.98-2.80(\mathrm{~m}, 1 \mathrm{H})$, 2.45-2.25 (m, 1H), 2.24-1.95 (m, 3H), 1.94-1.75 (m, 2H), 1.75-1.28 (m, 12H); ${ }^{13} \mathrm{C} N M R$ $\left(75 \mathrm{MHz}, \mathrm{CDCl}_{3}\right) \delta$ (rotamers) 174.5, 174.2, 157.0, 154.4, 153.5, 134.9, 134.8, 127.3, $127.1,126.9,121.0,120.8,110.6,80.3,80.1,59.9,59.3,58.6,58.1,55.7,52.6,52.4$, $37.0,36.4,34.7,34.4,34.2,34.1,33.0,32.2,28.8,28.7,27.4,27.3,26.5$; $[\alpha]_{D}-25.0^{\circ}$ (c 1.35, $\mathrm{CHCl}_{3}$ ); $\mathrm{HRMS}$ for $\mathrm{C}_{22} \mathrm{H}_{31} \mathrm{NO}_{5}$ calculated $\left(\mathrm{H}^{+}\right)$389.220223, found 389.222015. 


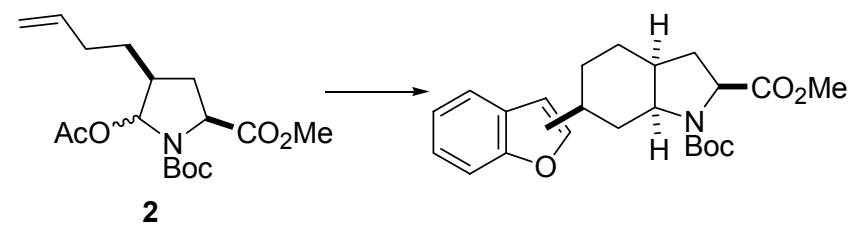

(2S,3aS,6S,7aS)-6-(2/3-Benzofuranyl)-2,3,3a,4,5,7a-hexahydro-indole-1,2-

dicarboxylic acid 1-tert-butyl ester 2-methyl ester (Entry 6, table 1). Same procedure

as for $\underline{5}$, except that the reaction was done in benzofuran at $-25^{\circ} \mathrm{C}(50 \%) ;{ }^{1} \mathrm{H}$ NMR $(400$

Deleted: 9

$\left.\mathrm{MHz}, \mathrm{CDCl}_{3}\right) \delta$ (mixture of regioisomers and rotamers) 7.63-7.05 $(\mathrm{m}, 4 \mathrm{H}), 6.82-6.68(\mathrm{~m}$, $1 \mathrm{H}), 4.38-4.19(\mathrm{~m}, 1 \mathrm{H}), 4.18-4.05(\mathrm{~m}, 1 \mathrm{H}), 3.91-3.66(\mathrm{~m}, 3 \mathrm{H}), 2.65-2.52(\mathrm{~m}, 1 \mathrm{H}), 2.50-$ $2.28(\mathrm{~m}, 2 \mathrm{H}), 2.28-2.02(\mathrm{~m}, 2 \mathrm{H}), 2.01-1.30(\mathrm{~m}, 14 \mathrm{H}) ;{ }^{13} \mathrm{C} \mathrm{NMR}\left(100 \mathrm{MHz}, \mathrm{CDCl}_{3}\right) \delta$ (mixture of regioisomers and rotamers) 174.4, 174.2, 154.3, 153.3, 145.6, 145.5, 144.9, $144.8,139.8,124.8,124.7,124.0,121.8,119.6,119.3,119.1,111.5,111.4,109.6$, 106.9, 106.7, 105.2, 80.2, 59.8, 59.3, 58.4, 58.1, 52.6, 52.4, 39.6, 39.5, 36.3, 36.2, 36.1, $34.7,32.9,32.2,28.8,28.7,28.0,27.7,26.5,26.3,19.8$; HRMS for $\mathrm{C}_{23} \mathrm{H}_{29} \mathrm{NO}_{5}$ calculated $\left(\mathrm{M}^{+}\right)$399.204573, found 399.205404.

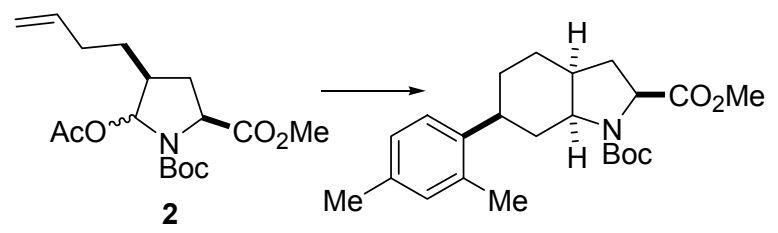

(2S,3aS,6S,7aS)-6-(2,4-Dimethyl-phenyl)-octahydro-indole-1,2-dicarboxylic acid 1tert-butyl ester 2-methyl ester (Entry 7, table 1). Same procedure as for $\underline{\mathbf{5}}$, except that the reaction was done in $m$-xylene at $-45^{\circ} \mathrm{C}(64 \%) ;{ }^{1} \mathrm{H}$ NMR $\left(400 \mathrm{MHz}, \mathrm{CDCl}_{3}\right) \delta$ (rotamers) 7.17-7.05 (m, 1H), 7.02-1.95 (m, 2H), 4.36-4.18 (m, 1H), 4.07-3.88 (m, 1H), 3.80-3.65 (m, 3H), 2.98-2.86 (m, 0.4H), 2.68-2.58 (m, 0.6H), 2.50-1.98 $(\mathrm{m}, 11 \mathrm{H}), 1.93-$ $1.77(\mathrm{~m}, 2 \mathrm{H}), 1.70-1.30(\mathrm{~m}, 11 \mathrm{H}) ;{ }^{13} \mathrm{C}$ NMR $\left(100 \mathrm{MHz}, \mathrm{CDCl}_{3}\right) \delta$ (rotamers) 174.5, $174.2,154.3,153.5,141.6,136.5,135.7,135.5,135.4,131.6,131.5,127.3,127.1$, $126.3,126.1,125.7,80.2,80.1,59.8,59.3,58.6,58.2,52.6,52.4,39.1$, 38.8, 37.4, 37.2, 
36.9, 36.4, 36.3, 35.1, 34.9, 33.0, 32.2, 28.8, 28.7, 28.0, 26.6, 21.3, 19.7, 19.5; $[\alpha]_{\mathrm{D}}-22^{\circ}$ (c 1.2, $\mathrm{CHCl}_{3}$ ); HRMS for $\mathrm{C}_{23} \mathrm{H}_{34} \mathrm{NO}_{4}$ calculated $\left(\mathrm{M}+\mathrm{H}^{+}\right.$) 388.248784, found 388.247364 .

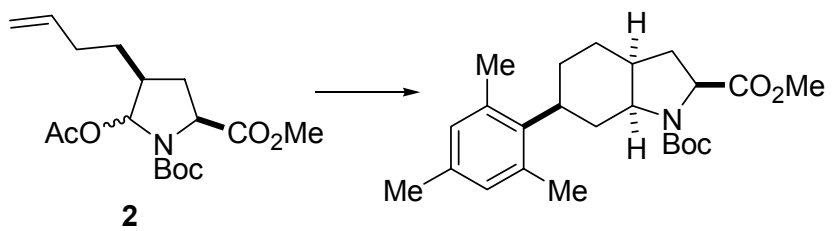

(2S,3aS,6S,7aS)-6-(2,4,6-Trimethyl-phenyl)-octahydro-indole-1,2-dicarboxylic acid 1-tert-butyl ester 2-methyl ester (Entry 8, table 1). Same procedure as for $\underline{5}$, except Deleted: 9 that the reaction was done in mesitylene at $-40^{\circ} \mathrm{C}(78 \%) ;{ }^{1} \mathrm{H} \mathrm{NMR}\left(300 \mathrm{MHz}, \mathrm{CDCl}_{3}\right) \delta$ (rotamers) 6.84-6.78 (m, 2H), 4.35-4.23 (m, 1H), 4.08-3.80 (m, 1H), $3.79(\mathrm{~s}, 1.5 \mathrm{H}), 3.76$ (s, 1.5H), 2.96-2.85 (m, 1H), 2.44-1.91 (m, 15H), 1.87-1.79 (m, 2H), 1.53-1.39 (m, 10H); ${ }^{13} \mathrm{C} \mathrm{NMR}\left(75 \mathrm{MHz}, \mathrm{CDCl}_{3}\right) \delta$ (rotamers) $174.5,174.2,154.3,153.5,139.1,139.0,135.6$, $135.4,80.2$, 80.1, 59.8, 59.3, 58.6, 58.2, 52.6, 52.4, 38.7, 38.4, 37.1, 36.4, 33.2, 32.4, 32.0, 31.4, 28.8, 28.7, 27.2, 27.1, 25.0, 24.8, 21.0; $[\alpha]_{\mathrm{D}}-31.7^{\circ}$ (c 1.06, $\mathrm{CHCl}_{3}$ ); $\mathrm{HRMS}$ for $\mathrm{C}_{24} \mathrm{H}_{35} \mathrm{NO}_{4}$ calculated $\left(\mathrm{M}^{+}\right) 401.256609$, found 401.257421 .

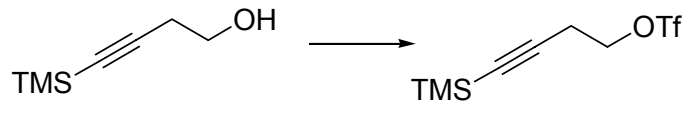

10

Trifluoro-methanesulfonic acid 4-trimethylsilanyl-but-3-ynyl ester (10). To a Deleted: 16 solution of pyridine $(5.63 \mathrm{~mL}, 69.7 \mathrm{mmol})$ in $\mathrm{CH}_{2} \mathrm{Cl}_{2}(70 \mathrm{~mL})$ at $-78^{\circ} \mathrm{C}$, was added $\mathrm{Tf}_{2} \mathrm{O}$ $(9.78 \mathrm{~mL}, 58.1 \mathrm{mmol})$, the mixture was stirred for $15 \mathrm{~min}$. and the alcohol $(8.26 \mathrm{~g}, 58.1$ mmol) was added dropwise. After stirring for $20 \mathrm{~min}$., the mixture was diluted with $\mathrm{CH}_{2} \mathrm{Cl}_{2}$, washed with ice cooled $\mathrm{HCl} 1 \mathrm{~N}(40 \mathrm{~mL})$, water and dried over $\mathrm{Na}_{2} \mathrm{SO}_{4}$. The solvent was removed under vacuum (without heating) and the resulting oil was purified on a small pad of silica gel (Hexanes/ $\mathrm{Et}_{2} \mathrm{O} 5: 1$ ) to give the corresponding triflate $\underline{10}$ Deleted: 16 (11.7 g, 74\%) as a light brown oil; ${ }^{1} \mathrm{H}$ NMR $\left(300 \mathrm{MHz}, \mathrm{CDCl}_{3}\right) \delta 4.56(\mathrm{t}, 2 \mathrm{H}, \mathrm{J}=6.8 \mathrm{~Hz})$, 
$2.76(\mathrm{t}, 2 \mathrm{H}, \mathrm{J}=6.8 \mathrm{~Hz}), 0.15(\mathrm{~s}, 3 \mathrm{H}) ;{ }^{13} \mathrm{C} \mathrm{NMR}\left(75 \mathrm{MHz}, \mathrm{CDCl}_{3}\right) \delta 120.6,116.3,98.4$, $88.6,73.6,21.0,-0.4$.

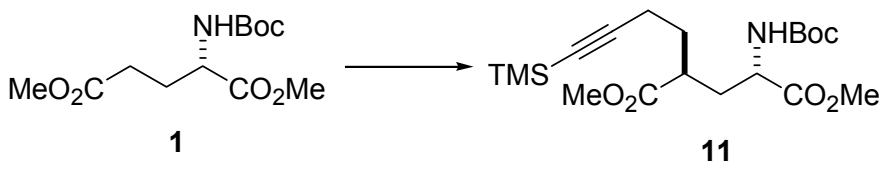

\section{(2S,4S)-2-tert-Butoxycarbonylamino-4-(4-trimethylsilanyl-but-3-ynyl)-pentanedioic} acid dimethyl ester (11). To a solution of $\mathrm{N}$-Boc-glutamic acid dimethylester $1(4.72 \mathrm{~g}$, $17,1 \mathrm{mmol})$ in THF $(100 \mathrm{~mL})$ at $-78^{\circ} \mathrm{C}$, was added LiHMDS $(36.0 \mathrm{~mL}, 1.0 \mathrm{~N}$ in THF), stirred for $45 \mathrm{~min}$. and a solution of the triflate $10(9.32 \mathrm{~g}, 34 \mathrm{mmol})$ in THF $(35 \mathrm{~mL})$ at $78^{\circ} \mathrm{C}$ was added dropwise via canula. After stirring for $20 \mathrm{~min}$., the solution was quenched with $\mathrm{NH}_{4} \mathrm{Cl}(2 \mathrm{~N})$, warmed up to $\mathrm{RT}$ and concentrated under vacuum. The resulting aqueous solution was extracted with $\mathrm{CH}_{2} \mathrm{Cl}_{2}$, the combined organic extracts were washed with brine and dried over $\mathrm{Na}_{2} \mathrm{SO}_{4}$. The solvent was removed under vacuum and the resulting oil was purified by flash chromatography (EtOAc/Hexanes 1:9 to $15: 85)$ to give $11(4.44 \mathrm{~g}, 65 \%)$ as a colorless oil; ${ }^{1} \mathrm{H}$ NMR $\left(400 \mathrm{MHz}, \mathrm{CDCl}_{3}\right) \delta 5.00$ $4.88(\mathrm{~m}, 1 \mathrm{H}), 4.30-4.20(\mathrm{~m}, 1 \mathrm{H}), 3.68(\mathrm{~s}, 3 \mathrm{H}), 3.63(\mathrm{~s}, 3 \mathrm{H}), 2.58-2.47(\mathrm{~m}, 1 \mathrm{H}), 2.16(\mathrm{t}$, $2 \mathrm{H}, \mathrm{J}=7.3 \mathrm{~Hz}), 2.00-1.85(\mathrm{~m}, 2 \mathrm{H}), 1.83-1.75(\mathrm{~m}, 1 \mathrm{H}), 1.75-1.61(\mathrm{~m}, 1 \mathrm{H}), 1.39(\mathrm{~s}, 9 \mathrm{H})$, 0.09 (s, 9H); ${ }^{13} \mathrm{C}$ NMR $\left(100 \mathrm{MHz}, \mathrm{CDCl}_{3}\right) \delta 175.8,173.1,155.7,106.0,85.7,80.4,52.8$, $52.5,52.2,41.6,34.4,31.5,28.6,18.1,0.4 ;[\alpha]_{D}+3.2^{\circ}$ (c 1.5, $\mathrm{CHCl}_{3}$ ); FAB/MS for $\mathrm{C}_{19} \mathrm{H}_{34} \mathrm{NO}_{6} \mathrm{Si}$ calculated $\left(\mathrm{M}+\mathrm{H}^{+}\right)$400.2, found 400.2 .

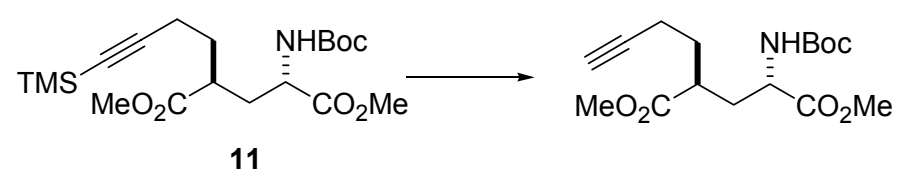

(2S,4S)-2-tert-Butoxycarbonylamino-4-but-3-ynyl-pentanedioic acid dimethyl ester. To a solution of the TMS derivative $11(4.00 \mathrm{~g}, 10.0 \mathrm{mmol})$ in THF $(50 \mathrm{~mL})$, was added $\mathrm{AcOH}(0.63 \mathrm{~mL}, 11.0 \mathrm{mmol})$ and a solution of TBAF $(11.0 \mathrm{~mL}, 1.0 \mathrm{M}$ in THF). After stirring at RT for 45 min., the solution was concentrated under vacuum and the

Deleted: 17

Deleted: 17

Deleted: 5

Deleted: 16 
resulting residue was purified by flash chromatography (EtOAc/Hexanes 25:75) to give the corresponding terminal alkyne $(2.88 \mathrm{~g}, 88 \%)$ as a colorless oil; ${ }^{1} \mathrm{H}$ NMR $(400 \mathrm{MHz}$, $\left.\mathrm{CDCl}_{3}\right) \delta 4.98(\mathrm{~d}, 1 \mathrm{H}, \mathrm{J}=8.7 \mathrm{~Hz}), 4.35-4.30(\mathrm{~m}, 1 \mathrm{H}), 3.71(\mathrm{~s}, 3 \mathrm{H}), 3.66(\mathrm{~s}, 3 \mathrm{H}), 2.65-2.61$ $(\mathrm{m}, 1 \mathrm{H}), 2.22-2.15(\mathrm{~m}, 2 \mathrm{H}), 2.02-1.90(\mathrm{~m}, 3 \mathrm{H}), 1.88-1.78(\mathrm{~m}, 1 \mathrm{H}), 1.77-1.68(\mathrm{~m}, 1 \mathrm{H})$, 1.41 (s, 9H); ${ }^{13} \mathrm{C}$ NMR $\left(100 \mathrm{MHz}, \mathrm{CDCl}_{3}\right) \delta 175.3,172.5,155.1,82.7,79.9,69.0,52.2$, $51.9,51.7,40.8,34.0,30.7,28.1,16.1 ;[\alpha]_{\mathrm{D}}+7.9^{\circ}$ (c $0.90, \mathrm{CHCl}_{3}$ ); FAB/MS for $\mathrm{C}_{16} \mathrm{H}_{26} \mathrm{NO}_{6}$ calculated $\left(\mathrm{M}+\mathrm{H}^{+}\right)$328.2, found 328.2.

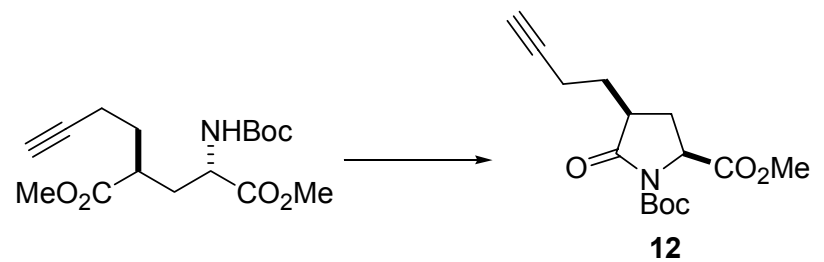

(2S,4S)-4-But-3-ynyl-5-oxo-pyrrolidine-1,2-dicarboxylic acid 1-tert-butyl ester 2methyl ester (12). To a solution of the of $\mathrm{N}$-Boc-glutamic acid dimethylester derivative $(2.50 \mathrm{~g}, 7.64 \mathrm{mmol})$ in $\mathrm{CH}_{2} \mathrm{Cl}_{2}(30 \mathrm{~mL})$, was added TFA $(5.6 \mathrm{~mL})$. After stirring at RT until no trace of starting material, the solution was concentrated under vacuum, the resulting oil was dissolved in toluene $(100 \mathrm{~mL})$, heated at reflux for $1.5 \mathrm{~h}$ and the solution was concentrated under vacuum. To a solution of the lactams obtained in $\mathrm{CH}_{2} \mathrm{Cl}_{2}$ (30 $\mathrm{mL})$, was added successively $\mathrm{Et}_{3} \mathrm{~N}(3.20 \mathrm{~mL}, 22.9 \mathrm{mmol}), \mathrm{Boc}_{2} \mathrm{O}(2.50 \mathrm{~g}, 11.5 \mathrm{mmol})$ followed by DMAP (cat.). After stirring overnight, the solution was quenched with $\mathrm{NH}_{4} \mathrm{Cl}$ $(2 \mathrm{~N})$, the aqueous layer was extracted with $\mathrm{CH}_{2} \mathrm{Cl}_{2}$, the combined organic extracts were washed with brine, dried over $\mathrm{Na}_{2} \mathrm{SO}_{4}$ and concentrated under vacuum. The crude oil obtained was purified by flash chromatography (EtOAc/Hexanes 15:85 to 30:70) to give compounds $12(1.80 \mathrm{~g}, 80 \%)$ as a colorless oil; ${ }^{1} \mathrm{H}$ NMR $\left(400 \mathrm{MHz}, \mathrm{CDCl}_{3}\right) \delta 4.47$ (t, $1 \mathrm{H}$, $J=8.2 \mathrm{~Hz}), 3.74(\mathrm{~s}, 3 \mathrm{H}), 2.75-2.65(\mathrm{~m}, 1 \mathrm{H}), 2.60-2.50(\mathrm{~m}, 1 \mathrm{H}), 2.42-2.31(\mathrm{~m}, 1 \mathrm{H}), 2.28-$ $2.18(\mathrm{~m}, 1 \mathrm{H}), 2.13-2.04(\mathrm{~m}, 1 \mathrm{H}), 1.94(\mathrm{t}, 1 \mathrm{H}, \mathrm{J}=2.6 \mathrm{~Hz}), 1.70-1.50(\mathrm{~m}, 2 \mathrm{H}), 1.45(\mathrm{~s}, 9 \mathrm{H})$; ${ }^{13} \mathrm{C}$ NMR $\left(100 \mathrm{MHz}, \mathrm{CDCl}_{3}\right) \delta 175.0,172.3,149.6,84.1,83.2,69.9,57.7,52.9,41.8$, $30.1,28.2,16.7$; $[\alpha]_{D}-10.7^{\circ}$ (c $1.00, \mathrm{CHCl}_{3}$ ); HRMS for $\mathrm{C}_{15} \mathrm{H}_{22} \mathrm{NO}_{5}$ calculated $\left(\mathrm{M}+\mathrm{H}^{+}\right.$) 296.149798 , found 296.151085 . 


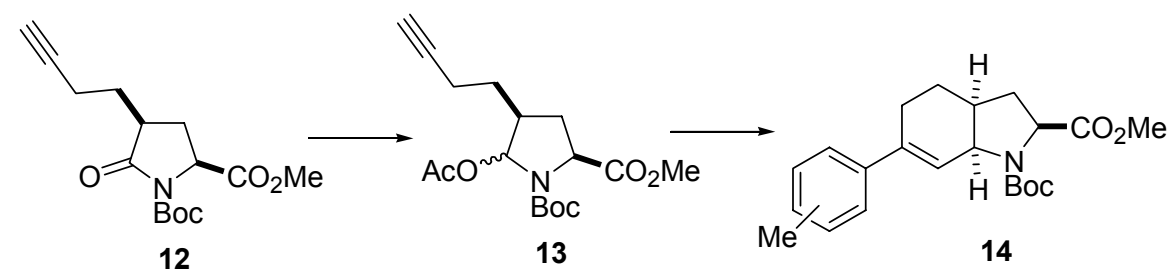

(2S,3aS,7aS)-6-(o/p-Tolyl)-2,3,3a,4,5,7a-hexahydro-indole-1,2-dicarboxylic acid 1tert-butyl ester 2-methyl ester (14). Same procedure as 2 for reduction/acetylation (94\%) and as compound 5 for cyclization $(69 \%) ;{ }^{1} \mathrm{H}$ NMR $\left(400 \mathrm{MHz}, \mathrm{CDCl}_{3}\right) \delta$ (mixture of regioisomers and rotamers) 7.35-7.00 (m, 4H), $6.21(\mathrm{~s}, 0.25 \mathrm{H}), 6.08(\mathrm{~s}, 0.25 \mathrm{H}), 5.78$ (s, 0.25H), $5.65(\mathrm{~s}, 0.25 \mathrm{H}), 4.60-4.50(\mathrm{~m}, 0.5 \mathrm{H}), 4.48-4.40(\mathrm{~m}, 0.5 \mathrm{H}), 4.38-4.20(\mathrm{~m}, 1 \mathrm{H})$, 3.77-3.62 (m, 3H), 2.60-2.43 (m, 1H), 2.40-1.80 (m, 9H), 1.56-1.38 (m, 9H); ${ }^{13} \mathrm{C} \mathrm{NMR}$ $\left(100 \mathrm{MHz}, \mathrm{CDCl}_{3}\right) \delta$ (mixture of regioisomers and rotamers) 174.0, 173.9, 154.6, 154.2, $154.0,143.7,138.9,138.6,135.5,135.3,130.5,130.3,129.4,129.3,128.7,128.6$, $128.3,127.3,127.2,126.1,126.0,125.9,125.5,125.3,123.1,80.4,59.6,59.5,59.1$, $57.5,57.1,56.9,52.5,52.4,52.3,35.7,35.1,34.9,32.3,32.2,31.5,28.9,28.7,28.6$, 25.3, 25.1, 23.2, 22.9, 20.0, 19.9; HRMS for $\mathrm{C}_{22} \mathrm{H}_{29} \mathrm{NO}_{4}$ calculated $\left(\mathrm{M}^{+}\right)$372.217484, found 372.218648 .

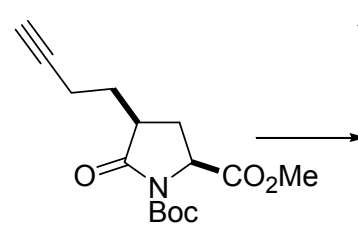

12

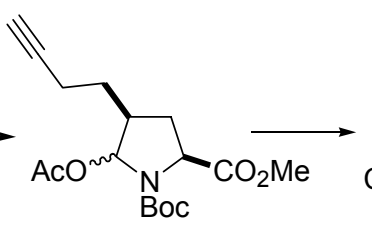

13<smiles>CC(=O)OC1C[C@H]2CCC(Cl)=C[C@H]2N1</smiles>

$16 a$

(2S,3aS,7aS)-6-Chloro-2,3,3a,4,5,7a-hexahydro-indole-1,2-dicarboxylic acid 1-tertbutyl ester 2-methyl ester (16a). Same procedure as 2 for reduction/acetylation (94\%). To a solution of the hemiaminal derivative $13(0.200 \mathrm{~g}, 0.589 \mathrm{mmol})$ in $\mathrm{CH}_{2} \mathrm{Cl}_{2}(5 \mathrm{~mL})$ at \begin{tabular}{|l|}
\hline Deleted: $22 \mathrm{a}$ \\
\hline Deleted: 6 \\
\hline Deleted: 19 \\
\hline
\end{tabular} $-78^{\circ} \mathrm{C}$, was added a solution of $\mathrm{SnCl}_{4}\left(0.77 \mathrm{~mL}, 1.0 \mathrm{M}\right.$ in $\left.\mathrm{CH}_{2} \mathrm{Cl}_{2}\right)$ dropwise. After stirring for 10 min., the solution was quenched with $\mathrm{NaHCO}_{3}$ (sat.), warmed up to RT, filtered on a small celite pad, the filtrated was washed with brine, dried over $\mathrm{Na}_{2} \mathrm{SO}_{4}$ and

\begin{tabular}{|l|}
\hline Deleted: 20 \\
Deleted: 6 \\
Deleted: 9
\end{tabular}


concentrated under vacuum. The crude oil obtained was purified by flash chromatography (EtOAc/Hexanes $10: 90$ to $15: 85)$ to give $16 \mathrm{a}(0.106 \mathrm{~g}, 57 \%)$ as a colorless oil; ${ }^{1} \mathrm{H}$ NMR $\left(400 \mathrm{MHz}, \mathrm{CDCl}_{3}\right) \delta$ (rotamers) 6.03 (broad s, 0.5H), 5.90 (broad $\mathrm{s}, 0.5 \mathrm{H}$ ), 4.42 (broad s, 0.5), 4.33 (broad s, 0.5H), 4.30-4.10 (m, 1H), $3.70(\mathrm{~s}, 3 \mathrm{H}), 2.50-$ $2.27(\mathrm{~m}, 2 \mathrm{H}), 2.26-2.08(\mathrm{~m}, 2 \mathrm{H}), 1.97-1.70(\mathrm{~m}, 3 \mathrm{H}), 1.46(\mathrm{~s}, 4.5 \mathrm{H}), 1.39(\mathrm{~s}, 4.5 \mathrm{H}) ;{ }^{13} \mathrm{C}$ NMR $\left(100 \mathrm{MHz}, \mathrm{CDCl}_{3}\right) \delta$ (rotamers) 173.6, 173.2, 154.3, 153.9, 132.9, 132.5, 124.4, 124.2, 80.8, 80.7, 59.4, 58.9, 57.8, 57.6, 52.6, 52.4, 35.2, 34.5, 32.0, 31.2, 28.8, 28.6, 28.3, 28.2, 28.1, 23.5, 23.4; $[\alpha]_{\mathrm{D}}+31.8^{\circ}$ (c 1.08, $\mathrm{CHCl}_{3}$ ); HRMS for $\mathrm{C}_{15} \mathrm{H}_{21} \mathrm{NO}_{4} \mathrm{Cl}$ calculated $\left(\mathrm{M}-\mathrm{H}^{-}\right)$314.115911, found 314.116090.

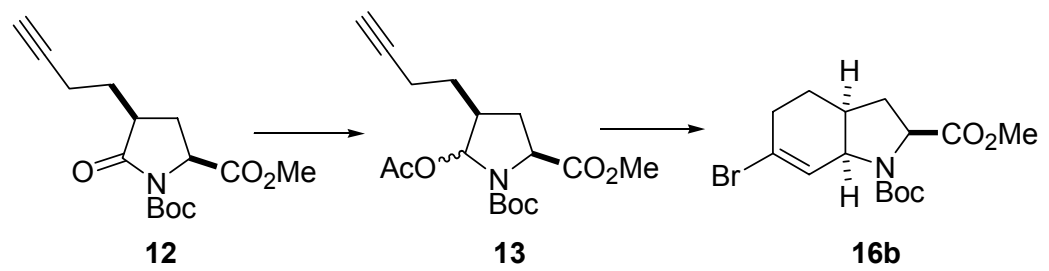

(2S,3aS,7aS)-6-Bromo-2,3,3a,4,5,7a-hexahydro-indole-1,2-dicarboxylic acid 1-tertbutyl ester 2-methyl ester (16b). Same procedure as 2 for reduction/acetylation (94\%) and same procedure as $16 \mathrm{a}$ for cyclization $(80 \%) ;{ }^{1} \mathrm{H}$ NMR $\left(400 \mathrm{MHz}, \mathrm{CDCl}_{3}\right) \delta$ (rotamers) 6.26 (broad s, 0.5H), 6.13 (broad s, 0.5H), 2.41-4.33 (broad s, $0.5 \mathrm{H}$ ), 4.33$4.10(\mathrm{~m}, 1.5 \mathrm{H}), 3.70(\mathrm{~s}, 3 \mathrm{H}), 2.50-2.22(\mathrm{~m}, 3 \mathrm{H}), 2.21-2.08(\mathrm{~m}, 1 \mathrm{H}), 1.98-1.67(\mathrm{~m}, 3 \mathrm{H})$, $1.45(\mathrm{~s}, 4.5 \mathrm{H}), 1.38(\mathrm{~s}, 4.5 \mathrm{H}) ;{ }^{13} \mathrm{C} \mathrm{NMR}\left(100 \mathrm{MHz}, \mathrm{CDCl}_{3}\right) \delta$ (rotamers) 173.6, 173.2, 154.2, 153.8, 128.5, 128.4, 123.2, 122.8, 80.9, 80.7, 59.4, 58.9, 58.6, 58.3, 57.6, 52.6, $52.5,35.1,34.5,34.3,32.0,31.2,30.6,30.5,28.8,28.6,28.3,24.1,24.0,23.4 ;[\alpha]_{D}$ $+53.6^{\circ}$ (c 1.03, $\mathrm{CHCl}_{3}$ ); $\mathrm{HRMS}$ for $\mathrm{C}_{15} \mathrm{H}_{21} \mathrm{NO}_{4} \mathrm{Br}$ calculated $\left(\mathrm{M}-\mathrm{H}^{-}\right) 358.065395$, found

358.064219 .

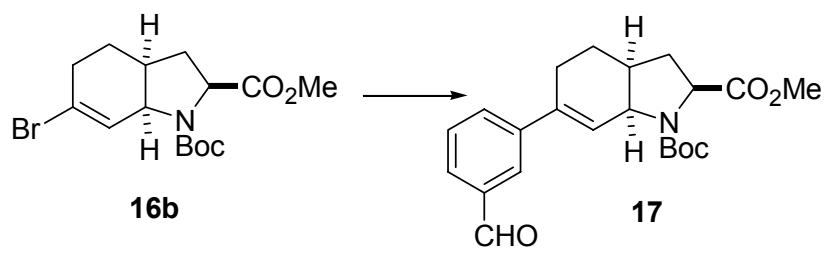

Deleted: 22a

\begin{tabular}{|l|}
\hline Deleted: 22b \\
\hline Deleted: 6 \\
\hline Deleted: 22a \\
\hline
\end{tabular}


(2S,3aS,7aS)-6-(3-Formyl-phenyl)-2,3,3a,4,5,7a-hexahydro-indole-1,2-dicarboxylic acid 1-tert-butyl ester 2-methyl ester (17). Same procedure as $21(70 \%) ;{ }^{1} \mathrm{H}$ NMR $\left(400 \mathrm{MHz}, \mathrm{CDCl}_{3}\right) \delta$ (rotamers) $10.0(\mathrm{~s}, 1 \mathrm{H}), 7.90-7.80(\mathrm{~m}, 1 \mathrm{H}), 7.78-7.57(\mathrm{~m}, 2 \mathrm{H}), 7.55-$ $7.38(\mathrm{~m}, 1 \mathrm{H}), 6.35(\mathrm{~s}, 0.6 \mathrm{H}), 6.19(\mathrm{~s}, 0.4 \mathrm{H}), 4.62-4.50(\mathrm{~m}, 0.6 \mathrm{H}), 4.49-4.40(\mathrm{~m}, 0.4 \mathrm{H})$, $4.30(\mathrm{t}, 0.4 \mathrm{H}, \mathrm{J}=8.2 \mathrm{~Hz}), 4.26(\mathrm{t}, 0.6 \mathrm{H}, \mathrm{J}=8.2 \mathrm{~Hz}), 3.69-3.65(\mathrm{~m}, 3 \mathrm{H}), 2.60-2.28(\mathrm{~m}, 3 \mathrm{H})$, 2.23-2.10 (m, $1 \mathrm{H}), 2.07-1.70(\mathrm{~m}, 4 \mathrm{H}), 1.50(\mathrm{~s}, 4.5 \mathrm{H}), 1.42(\mathrm{~s}, 4.5 \mathrm{H}) ;{ }^{13} \mathrm{C} N M R(100 \mathrm{MHz}$, $\left.\mathrm{CDCl}_{3}\right) \delta$ (rotamers) 192.9, 173.9, 154.2, 142.9, 136.8, 135.3, 132.1, 131.8, 129.5, $129.3,129.2$, 128.8, 127.2, 127.0, 126.4, 125.6, 125.4, 80.6, 59.4, 58.9, 57.5, 57.4, $56.1,52.5,52.4,35.7,35.0,32.2,31.4,28.9,28.7,22.9,22.7 ;[\alpha]_{D}+42.1^{\circ}$ (c 1.25, $\left.\mathrm{CHCl}_{3}\right)$; $\mathrm{FAB} / \mathrm{MS}$ for $\mathrm{C}_{22} \mathrm{H}_{28} \mathrm{NO}_{5}$ calculated $\left(\mathrm{M}+\mathrm{H}^{+}\right)$386.2, found 386.2.

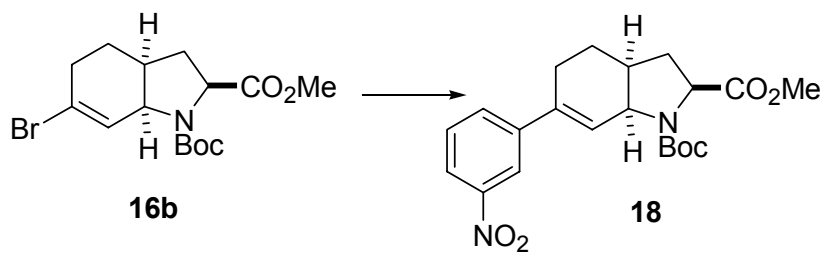

\section{(2S,3aS,7aS)-6-(3-Nitro-phenyl)-2,3,3a,4,5,7a-hexahydro-indole-1,2-dicarboxylic} acid 1-tert-butyl ester 2-methyl ester (18). Same procedure as 21 (73\%); ${ }^{1} \mathrm{H}$ NMR $\left(400 \mathrm{MHz}, \mathrm{CDCl}_{3}\right) \delta$ (rotamers) $8.20(\mathrm{~s}, 1 \mathrm{H}), 8.10-8.03(\mathrm{~m}, 1 \mathrm{H}), 7.78-7.63(\mathrm{~m}, 1 \mathrm{H}), 7.48-$ $7.39(\mathrm{~m}, 1 \mathrm{H}), 6.38(\mathrm{~s}, 0.6 \mathrm{H}), 6.22(\mathrm{~s}, 0.4 \mathrm{H}), 4.60-4.52(\mathrm{~m}, 0.6 \mathrm{H}), 4.50-4.41(\mathrm{~m}, 0.4 \mathrm{H})$, $4.31(\mathrm{t}, 0.4 \mathrm{H}, \mathrm{J}=8.3 \mathrm{~Hz}), 4.25(\mathrm{t}, 0.6 \mathrm{H}, \mathrm{J}=8.3 \mathrm{~Hz}), 3.67(\mathrm{~s}, 3 \mathrm{H}), 2.60-2.30(\mathrm{~m}, 3 \mathrm{H}), 2.27-$ $2.17(\mathrm{~m}, 1 \mathrm{H}), 2.02-1.75(\mathrm{~m}, 3 \mathrm{H}), 1.50(\mathrm{~s}, 4.5 \mathrm{H}), 1.43(\mathrm{~s}, 4.5 \mathrm{H}) ;{ }^{13} \mathrm{C} \mathrm{NMR}(100 \mathrm{MHz}$, $\left.\mathrm{CDCl}_{3}\right) \delta$ (rotamers) $173.9,173.6,154.2,148.7,143.7,135.2,134.5,132.1,131.6$, 129.7, 129.5, 127.6, 126.7, 126.5, 122.3, 120.8, 80.7, 60.8, 59.4, 58.9, 57.4, 57.2, 56.2, 52.5, 52.4, 34.9, 32.1, 31.3, 28.9, 28.7, 22.9, 22.8; $[\alpha]_{\mathrm{D}}+70.6^{\circ}$ (c 1.42, $\mathrm{CHCl}_{3}$ ); HRMS for $\mathrm{C}_{10} \mathrm{H}_{26} \mathrm{~N}_{2} \mathrm{O}_{6}$ calculated $\left(\mathrm{M}^{+}\right)$402.179087, found 402.179414.
Deleted: 23

Deleted: 27

Deleted: 24

Deleted: 27 
<smiles>CC(=O)C1CC2CCC(/C=C/c3ccccc3)=C[C@@H]2[NH2+][C@H]1C(=O)O</smiles>

(2S,3aS,7aS)-6-(E-Styryl)-2,3,3a,4,5,7a-hexahydro-indole-1,2-dicarboxylic acid 1tert-butyl ester 2-methyl ester (19). Same procedure as 21 (81\%); ${ }^{1} \mathrm{H}$ NMR $(400 \mathrm{MHz}$, $\left.\mathrm{CDCl}_{3}\right) \delta$ (rotamers) 7.42-7.10 (m, 5H), $6.80(\mathrm{~s}, 0.4 \mathrm{H}), 6.78(\mathrm{~s}, 0.6 \mathrm{H}), 6.50-6.42(\mathrm{~m}, 1 \mathrm{H})$, \begin{tabular}{|l|}
\hline Deleted: 25 \\
\hline Deleted: 27
\end{tabular} $6.04(\mathrm{~s}, 0.6 \mathrm{H}), 5.90(\mathrm{~s}, 0.4 \mathrm{H}), 4.47-4.43(\mathrm{~m}, 0.4 \mathrm{H}), 4.42-4.37(\mathrm{~m}, 0.6 \mathrm{H}), 4.30(\mathrm{t}, 0.4 \mathrm{H}$, $J=8.5 \mathrm{~Hz}), 4.23(\mathrm{t}, 0.6 \mathrm{H}, J=8.5 \mathrm{~Hz}), 3.75-3.62(\mathrm{~m}, 3 \mathrm{H}), 2.58-2.28(\mathrm{~m}, 2 \mathrm{H}), 2.23-2.08(\mathrm{~m}$, $2 \mathrm{H}), 1.98-1.75(\mathrm{~m}, 4 \mathrm{H}), 1.56-1.38(\mathrm{~m}, 9 \mathrm{H}) ;{ }^{13} \mathrm{C} \mathrm{NMR}\left(100 \mathrm{MHz}, \mathrm{CDCl}_{3}\right) \delta$ (rotamers) $173.9,146.9,137.9,135.4,135.0,132.2,132.1,129.8,129.0,128.7,127.9,127.6$, $127.2,80.8,80.5,59.6,59.0,57.6,57.4,56.2,52.5,52.4,36.3,35.5,32.2,31.5,28.9$, 28.7, 22.5, 19.8; $[\alpha]_{D}+48^{\circ}$ (c $\left.0.75, \mathrm{CHCl}_{3}\right)$; $\mathrm{FAB} / \mathrm{MS}$ for $\mathrm{C}_{23} \mathrm{H}_{30} \mathrm{NO}_{4}$ calculated $\left(\mathrm{M}+\mathrm{H}^{+}\right)$ 384.2 , found 384.2 .

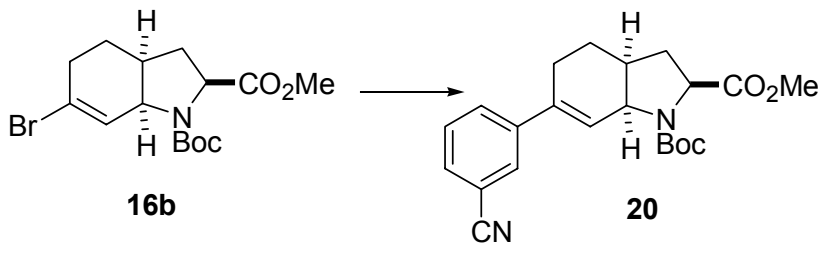

(2S,3aS,7aS)-6-(3-Cyano-phenyl)-2,3,3a,4,5,7a-hexahydro-indole-1,2-dicarboxylic acid 1-tert-butyl ester 2-methyl ester (20). Same procedure as $21(78 \%) ;{ }^{1} \mathrm{H}$ NMR $\left(400 \mathrm{MHz}, \mathrm{CDCl}_{3}\right) \delta$ (rotamers) 7.63-7.58 (m, 2H), 7.55-7.45 (m, 1H), 7.44-7.32 (m, $\left.1 \mathrm{H}\right)$, $6.30(\mathrm{~s}, 0.6 \mathrm{H}), 6.10(\mathrm{~s}, 0.4 \mathrm{H}), 4.58-4.48(\mathrm{~m}, 0.6 \mathrm{H}), 4.47-4.38(\mathrm{~m}, 0.4 \mathrm{H}), 4.32(\mathrm{t}, 0.4 \mathrm{H}$, $J=8.5 \mathrm{~Hz}), 4.25(\mathrm{t}, 0.6 \mathrm{H}, \mathrm{J}=8.5 \mathrm{~Hz}), 3.68-3.66(\mathrm{~m}, 3 \mathrm{H}), 2.60-2.27(\mathrm{~m}, 3 \mathrm{H}), 2.26-2.10(\mathrm{~m}$, $1 \mathrm{H}), 2.00-1.72(\mathrm{~m}, 3 \mathrm{H}), 1.53-1.30(\mathrm{~m}, 9 \mathrm{H}) ;{ }^{13} \mathrm{C} \mathrm{NMR}\left(100 \mathrm{MHz}, \mathrm{CDCl}_{3}\right) \delta$ (rotamers) $173.9,154.2$, 143.1, 134.6, 131.1, 130.9, 130.5, 130.4, 129.9, 129.6, 129.4, 126.4, 126.2, 119.4, 112.7, 80.7, 59.3, 58.8, 57.4, 57.2, 52.5, 52.4, 35.6, 34.9, 32.1, 31.3, 28.9, 28.7, 22.8; $[\alpha]_{D}+44.4^{\circ}$ (c 1.11, $\mathrm{CHCl}_{3}$ ); $\mathrm{FAB} / \mathrm{MS}$ for $\mathrm{C}_{22} \mathrm{H}_{27} \mathrm{~N}_{2} \mathrm{O}_{4}$ calculated $\left(\mathrm{M}+\mathrm{H}^{+}\right.$) 383.2, found 383.2 . 


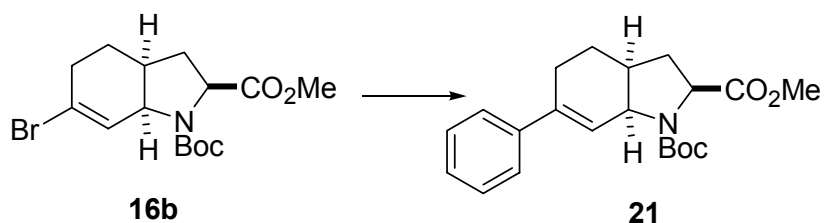

(2S,3aS,7aS)-6-Phenyl-2,3,3a,4,5,7a-hexahydro-indole-1,2-dicarboxylic acid 1-tertbutyl ester 2-methyl ester (21). To a solution of the bromide $16 \mathrm{~b}(0.080 \mathrm{~g}, 0.222$ mmol) and phenylboronic acid $(0.041 \mathrm{~g}, 0.333 \mathrm{mmol})$ in THF $(2 \mathrm{~mL})$, was added a Deleted: 27 Deleted: 22b solution of $\mathrm{Cs}_{2} \mathrm{CO}_{3}\left(0.88 \mathrm{~mL}, 1.0 \mathrm{M}\right.$ in $\left.\mathrm{H}_{2} \mathrm{O}\right)$ followed by $\mathrm{Pd}\left(\mathrm{PPh}_{3}\right)_{4}$ (cat.). After heating the solution at reflux for $4 \mathrm{~h}$, the mixture was concentrated under vacuum, the aqueous solution was extracted with $\mathrm{CH}_{2} \mathrm{Cl}_{2}$, the combined organic extracts were washed with brine and dried over $\mathrm{Na}_{2} \mathrm{SO}_{4}$. The solvent was removed under vacuum and the resulting oil was purified by flash chromatography (EtOAc/Hexanes $10: 90)$ to give 21 ( $0.067 \mathrm{~g}$, $84 \%$ ) as a colorless oil; ${ }^{1} \mathrm{H}$ NMR $\left(400 \mathrm{MHz}, \mathrm{CDCl}_{3}\right) \delta$ (rotamers) 6.45-6.15 (m, 4H), 6.25 $(\mathrm{s}, 0.5 \mathrm{H}), 6.10(\mathrm{~s}, 0.5 \mathrm{H}), 4.58-4.52(\mathrm{~m}, 0.5 \mathrm{H}), 4.50-4.40(\mathrm{~m}, 0.5 \mathrm{H}), 4.33(\mathrm{t}, 0.5 \mathrm{H}, J=8.4$ $\mathrm{Hz}), 4.25(\mathrm{t}, 0.5 \mathrm{H}, J=8.4 \mathrm{~Hz}), 3.75-3.61(\mathrm{~m}, 3 \mathrm{H}), 2.57-2.29(\mathrm{~m}, 3 \mathrm{H}), 2.28-2.12(\mathrm{~m}, 1 \mathrm{H})$, 2.08-1.75 (m, 4H), 1.56-1.30 (m, 9H); ${ }^{13} \mathrm{C}$ NMR (100 MHz, $\left.\mathrm{CDCl}_{3}\right) \delta$ (rotamers) 128.2, $128.0,127.1,126.9,125.5,123.3,79.9,59.0,56.9,51.9,34.5,31.6,28.4,28.2$, 22.4, 22.1; $[\alpha]_{D}+49.8^{\circ}$ (c 1.50, $\mathrm{CHCl}_{3}$ ); FAB/MS for $\mathrm{C}_{21} \mathrm{H}_{28} \mathrm{NO}_{4}$ calculated $\left(\mathrm{M}+\mathrm{H}^{+}\right.$) 358.2, found 358.2 .<smiles>CC(=O)OC(=O)c1cccc([N+](=O)C2C3C=C(Br)CCC3CC2C(OC(C)=O)C(C)=O)c1</smiles>

(2S,3aS,7aS)-6-(3-Hydroxy-phenyl)-2,3,3a,4,5,7a-hexahydro-indole-1,2dicarboxylic acid 1-tert-butyl ester 2-methyl ester (22). Same procedure as 21 (85\%); ${ }^{1} \mathrm{H}$ NMR $\left(400 \mathrm{MHz}, \mathrm{CDCl}_{3}\right) \delta$ (rotamers) 7.18-7.05 (m, 1H), 6.95-6.67 (m, 3H), 
$\mathrm{J}=8.2 \mathrm{~Hz}), 4.25(\mathrm{t}, 0.5 \mathrm{H}, \mathrm{J}=8.2 \mathrm{~Hz}), 3.68(\mathrm{~s}, 1.5 \mathrm{H}), 3.66(\mathrm{~s}, 1.5 \mathrm{H}), 2.58-2.10(\mathrm{~m}, 4 \mathrm{H})$, 2.03-1.68 (m, 3H), $1.50(\mathrm{~s}, 4.5 \mathrm{H}), 1.43(\mathrm{~s}, 4.5 \mathrm{H}) ;{ }^{13} \mathrm{C}$ NMR $\left(100 \mathrm{MHz}, \mathrm{CDCl}_{3}\right) \delta$ (rotamers) 174.1, 174.0, 157.3, 156.5, 154.9, 154.6, 143.4, 136.3, 130.3, 129.7, 129.6, $123.4,119.0,117.9,114.9,114.7,113.0,81.1,80.9,59.6,58.9,57.6,52.7,52.5,35.2$, $32.1,31.4,28.9,28.8,22.9,22.5,21.5 ;[\alpha]_{D}+21.7^{\circ}$ (c 1.08, $\mathrm{CHCl}_{3}$ ); HRMS for $\mathrm{C}_{21} \mathrm{H}_{27} \mathrm{NO}_{5}$ calculated $\left(\mathrm{M}^{+}\right)$373.188923, found 373.188356 .

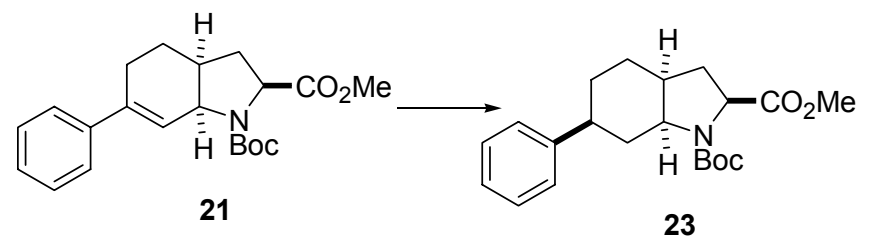

(2S,3aS,6S,7aS)-6-phenyl-octahydro-indole-1,2-dicarboxylic acid 1-tert-butyl ester 2-methyl ester (23). To a solution of $21(0.050 \mathrm{~g}, 0.140 \mathrm{mmol})$ in EtOAc $(2.5 \mathrm{~mL})$, was added $\mathrm{Pd} / \mathrm{C} 10 \%$ (cat.) and stirred under $1 \mathrm{~atm}$. of $\mathrm{H}_{2}$ for $24 \mathrm{~h}$. The catalyst was filtered through a celite pad, the filtrate was concentrated under vacuum and the resulting oil was purified by flash chromatography (EtOAc/Hexanes 0:100 to 10:90) to give $\underline{23}$ $(0.035 \mathrm{~g}, 70 \%)$ as a white solid crystallizing in hexanes; ${ }^{1} \mathrm{H} \mathrm{NMR}\left(400 \mathrm{MHz}, \mathrm{CDCl}_{3}\right) \delta$ (rotamers) 7.34-7.15 (m, 5H), 4.29 (dd, $0.6 \mathrm{H}, \mathrm{J}=7.7 \mathrm{~Hz}, \mathrm{~J}=9.9 \mathrm{~Hz}$ ), 4.24 (dd, $0.4 \mathrm{H}, \mathrm{J}=7.7$ $\mathrm{Hz}, J=9.9 \mathrm{~Hz}), 4.08-4.02(\mathrm{~m}, 0.6 \mathrm{H}), 3.95-3.90(\mathrm{~m}, 0.4 \mathrm{H}), 3.76(\mathrm{~s}, 1.5 \mathrm{H}), 3.74(\mathrm{~s}, 1.5 \mathrm{H})$, 2.53-2.30 (m, 2H), 2.25-2.00 (m, 2H), 1.90-1.79 (m, 2H), 1.75-1.50 (m, 4H), $1.43(\mathrm{~s}$, $4.5 \mathrm{H}), 1.39(\mathrm{~s}, 4.5 \mathrm{H}) ;{ }^{13} \mathrm{C} \mathrm{NMR}\left(100 \mathrm{MHz}, \mathrm{CDCl}_{3}\right) \delta$ (rotamers) 173.9, 153.0, 146.0, $128.3,128.1,126.7,126.0,125.8,79.7,59.3,58.7,57.8,57.5,52.0,51.8,41.6,41.4$, $36.3,35.6,35.1,34.8,32.4,31.6,28.6,28.3,28.1,27.8,25.9,25.7$; $[\alpha]_{D}-16.8^{\circ}$ (c 0.75, $\mathrm{CHCl}_{3}$ ); m.p.: $100-103^{\circ} \mathrm{C}$; HRMS for $\mathrm{C}_{21} \mathrm{H}_{30} \mathrm{NO}_{4}$ calculated $\left(\mathrm{M}+\mathrm{H}^{+}\right) 360.217484$, found 360.217267 .

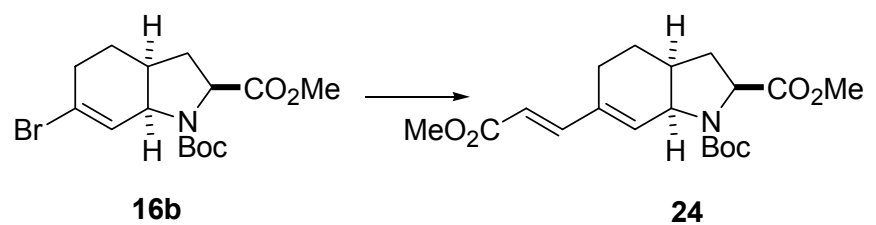


(2S,3aS,7aS)-6-(2-Methoxycarbonyl-vinyl)-2,3,3a,4,5,7a-hexahydro-indole-1,2-

dicarboxylic acid 1-tert-butyl ester 2-methyl ester (24). To a solution of the bromide $16 \mathrm{~b}(0.072 \mathrm{~g}, 0.200 \mathrm{mmol})$, methyl acrylate $(0.14 \mathrm{~mL}, 1.60 \mathrm{mmol})$ and $\mathrm{Et}_{3} \mathrm{~N}(0.11 \mathrm{~mL}$, $0.800 \mathrm{mmol})$ in $\mathrm{MeCN}(3 \mathrm{~mL})$, was added $\mathrm{P}(\mathrm{o} \text {-tolyl })_{3}(0.009 \mathrm{~g}, 0.03 \mathrm{mmol})$ and $\mathrm{Pd}(\mathrm{OAc})_{2}$ $(0.003 \mathrm{~g}, 0.01 \mathrm{mmol})$. After heating the solution at reflux for $12 \mathrm{~h}$ under argon, $\mathrm{P}(\mathrm{o} \text {-tolyl })_{3}$ $(0.009 \mathrm{~g}, 0.03 \mathrm{mmol})$ and $\mathrm{Pd}(\mathrm{OAc})_{2}(0.003 \mathrm{~g}, 0.01 \mathrm{mmol})$ were added and the heating was continue for another $12 \mathrm{~h}$. Finally, the solution was cooled down to $\mathrm{RT}, \mathrm{H}_{2} \mathrm{O}$ was added, the aqueous solution was extracted with $\mathrm{CH}_{2} \mathrm{Cl}_{2}$, the combined organic extracts were washed with brine and dried over $\mathrm{Na}_{2} \mathrm{SO}_{4}$. The solvent was removed under vacuum and the resulting oil was purified by flash chromatography (EtOAc/Hexanes $15: 85)$ to give $24(0.055 \mathrm{~g}, 75 \%)$ as a colorless oil; ${ }^{1} \mathrm{H}$ NMR $\left(400 \mathrm{MHz}, \mathrm{CDCl}_{3}\right) \delta 7.32(\mathrm{~d}$, $1 \mathrm{H}, J=15.9 \mathrm{~Hz}), 6.30(\mathrm{~s}, 0.6 \mathrm{H}), 6.18(\mathrm{~s}, 0.4 \mathrm{H}), 5.86-5.76(\mathrm{~m}, 1 \mathrm{H}), 4.51-4.42(\mathrm{~m}, 0.6 \mathrm{H})$, 4.40-4.33 (m, 0.4H), $4.27(\mathrm{t}, 0.4 \mathrm{H}, J=8.4 \mathrm{~Hz}), 4.22(\mathrm{t}, 0.6 \mathrm{H}, J=8.4 \mathrm{~Hz}), 3.74(\mathrm{~s}, 3 \mathrm{H}), 3.68$ (s, 3H), 2.58-2.41 (m, 1H), 2.22-1.97 (m, 3H), 1.88-1.70 (m, 3H), $1.50(\mathrm{~s}, 4.5 \mathrm{H}), 1.42(\mathrm{~s}$, $4.5 \mathrm{H}) ;{ }^{13} \mathrm{C}$ NMR $\left(100 \mathrm{MHz}, \mathrm{CDCl}_{3}\right) \delta 173.7,173.3,167.9,147.8,135.9,135.8,134.1$, 133.7, 117.2, 117.1, 80.8, 59.4, 58.9, 57.3, 57.1, 52.4, 51.9, 45.1, 36.0, 35.2, 32.1, 31.4, 28.9, 28.7, 22.2, 22.1, 19.5; $[\alpha]_{\mathrm{D}}+141^{\circ}$ (c $0.750, \mathrm{CHCl}_{3}$ ); HRMS for $\mathrm{C}_{19} \mathrm{H}_{27} \mathrm{NO}_{6}$ calculated $\left(\mathrm{M}^{+}\right) 365.183838$, found 365.183716 .

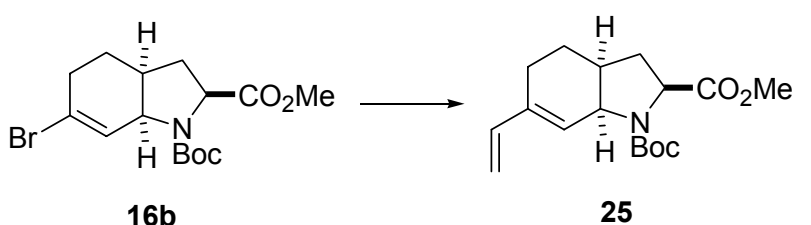

(2S,3aS,7aS)-6-Vinyl-2,3,3a,4,5,7a-hexahydro-indole-1,2-dicarboxylic acid 1-tertbutyl ester 2-methyl ester (25). To a solution of the $16 \mathrm{~b}(0.080 \mathrm{~g}, 0.222 \mathrm{mmol})$ and tributylvinyltin $(71 \mu \mathrm{L}, 0.244 \mathrm{mmol})$ in toluene $(2.5 \mathrm{~mL})$, was added $\mathrm{Pd}\left(\mathrm{PPh}_{3}\right)_{4}$ (cat.). After heating the solution at $100^{\circ} \mathrm{C}$ for $8 \mathrm{~h}$, the mixture was concentrated under vacuum and the resulting oil was purified by flash chromatography (EtOAc/Hexanes 10:90) to give $25(0.048 \mathrm{~g}, 71 \%)$ as a colorless oil; ${ }^{1} \mathrm{H}$ NMR $\left(400 \mathrm{MHz}, \mathrm{CDCl}_{3}\right) \delta$ (rotamers) 6.40-
Deleted: 30

Deleted: 22b

Deleted: 30
Deleted: 31

Deleted: 22b 
$6.33(\mathrm{~m}, 1 \mathrm{H}), 5.91(\mathrm{~s}, 0.6 \mathrm{H}), 5.78(\mathrm{~m}, 0.4 \mathrm{H}), 5.17-5.10(\mathrm{~m}, 1 \mathrm{H}), 5.03-4.96(\mathrm{~m}, 1 \mathrm{H}), 4.48-$ $4.41(\mathrm{~m}, 0.6 \mathrm{H}), 4.38-4.30(\mathrm{~m}, 0.4 \mathrm{H}), 4.28(\mathrm{t}, 0.4 \mathrm{H}, \mathrm{J}=8.3 \mathrm{~Hz}), 4.23(\mathrm{t}, 0.6 \mathrm{H}, \mathrm{J}=8.3 \mathrm{~Hz})$, $3.67(\mathrm{~s}, 3 \mathrm{H}), 2.50-2.35(\mathrm{~m}, 1 \mathrm{H}), 2.22-2.08(\mathrm{~m}, 2 \mathrm{H}), 2.07-1.90(\mathrm{~m}, 1 \mathrm{H}), 1.87-1.70(\mathrm{~m}$, $3 \mathrm{H}), 1.48(\mathrm{~s}, 4.5 \mathrm{H}), 1.41(\mathrm{~s}, 4.5 \mathrm{H}) ;{ }^{13} \mathrm{C}$ NMR $\left(100 \mathrm{MHz}, \mathrm{CDCl}_{3}\right) \delta$ (rotamers) 173.9, 154.2, 140.0, 135.6, 135.2, 128.1, 112.7, 112.4, 80.5, 59.5, 58.9, 57.4, 57.2, 52.5, 52.3, $36.3,35.5,32.2,31.4,28.9,28.7,22.3,19.0$; $[\alpha]_{D}+131^{\circ}$ (c 1.00, $\mathrm{CHCl}_{3}$ ); HRMS for $\mathrm{C}_{17} \mathrm{H}_{25} \mathrm{NO}_{4}$ calculated $\left(\mathrm{M}^{+}\right) 307.178359$, found 307.179443 . 
(C) 2004 American Chemical Society, Org. Lett., Hanessian ol040053b Supporting Info Page 22

\section{Universite th \\ de Montréal}

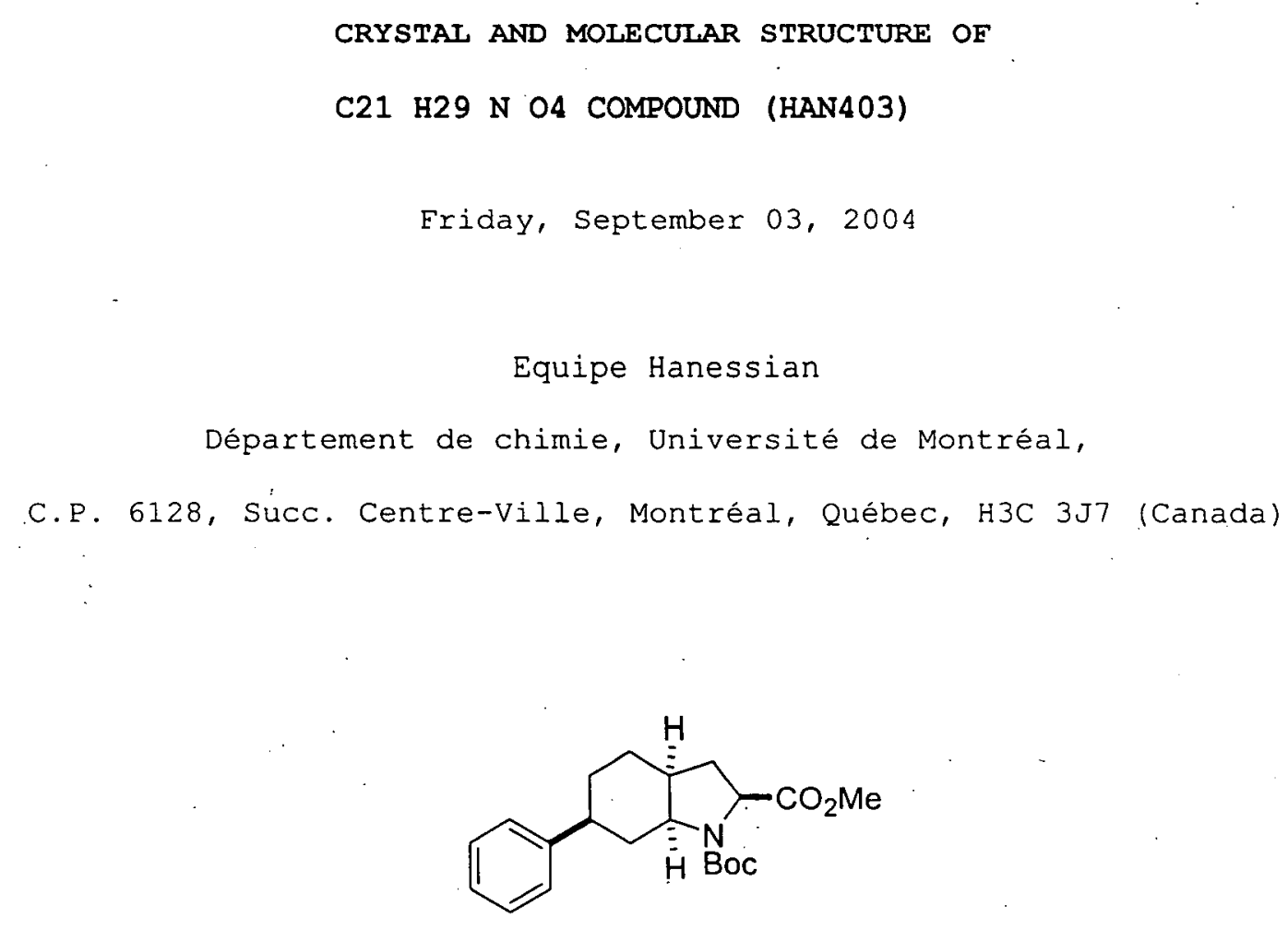

Structure solved and refined in the laboratory of X-ray diffraction Université de Montréal by Dr. Michel Simard. 
Table 1. Crystal data and structure refinement for C21 H29 $\mathrm{N} 04$.

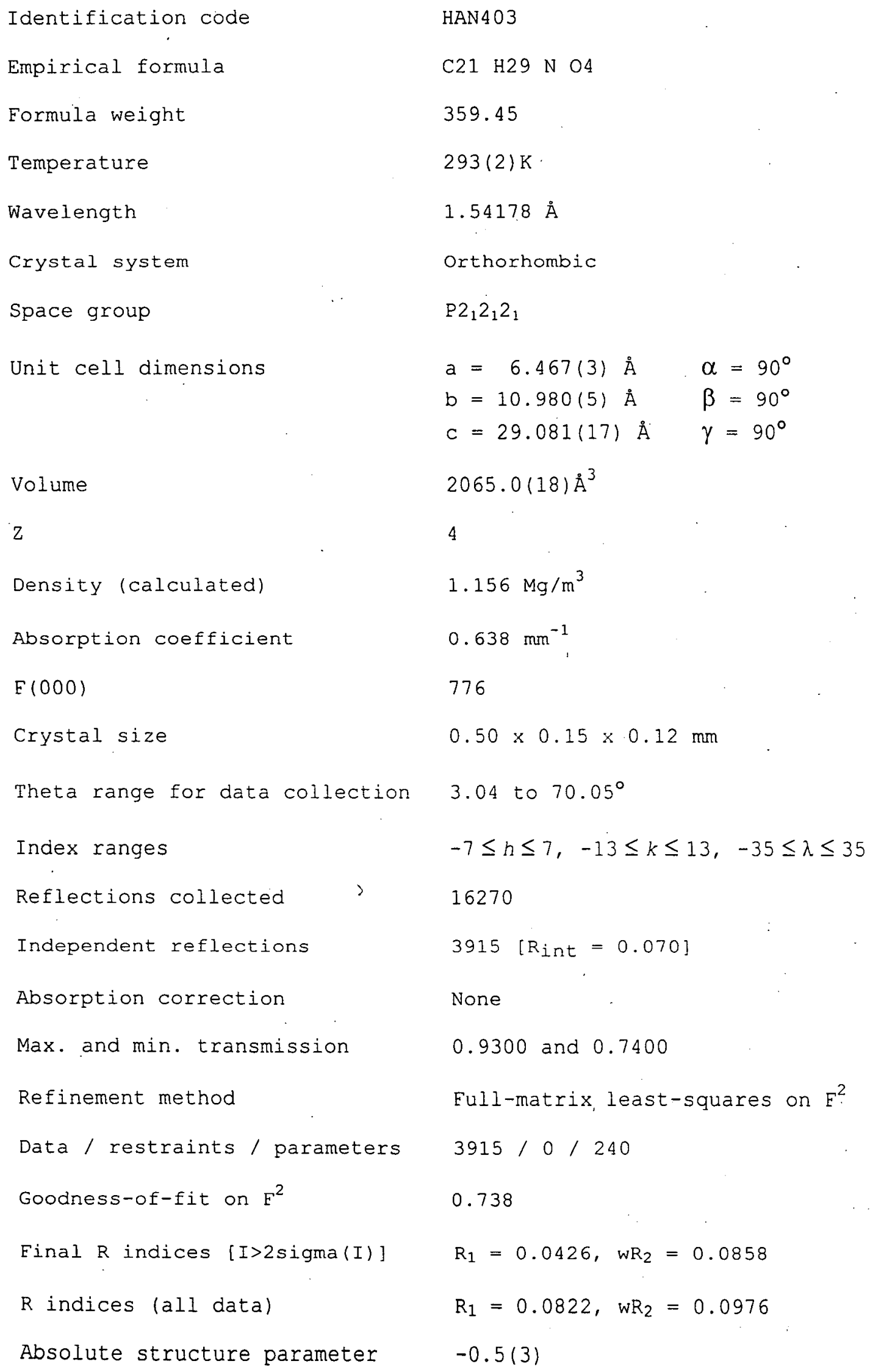


(C) 2004 American Chemical Society, Org. Lett., Hanessian ol040053b Supporting Info Page 24

$\begin{array}{ll}\text { Extinction coefficient } & 0.00322(19) \\ \text { Largest diff. peak and hole } & 0.124 \text { and }-0.141 \mathrm{e} / \hat{\mathrm{A}}^{3}\end{array}$


Table 5. Bond lengths [A] and angles [ $\left.{ }^{\circ}\right]$ for $\mathrm{C} 21$ H29 N O4.

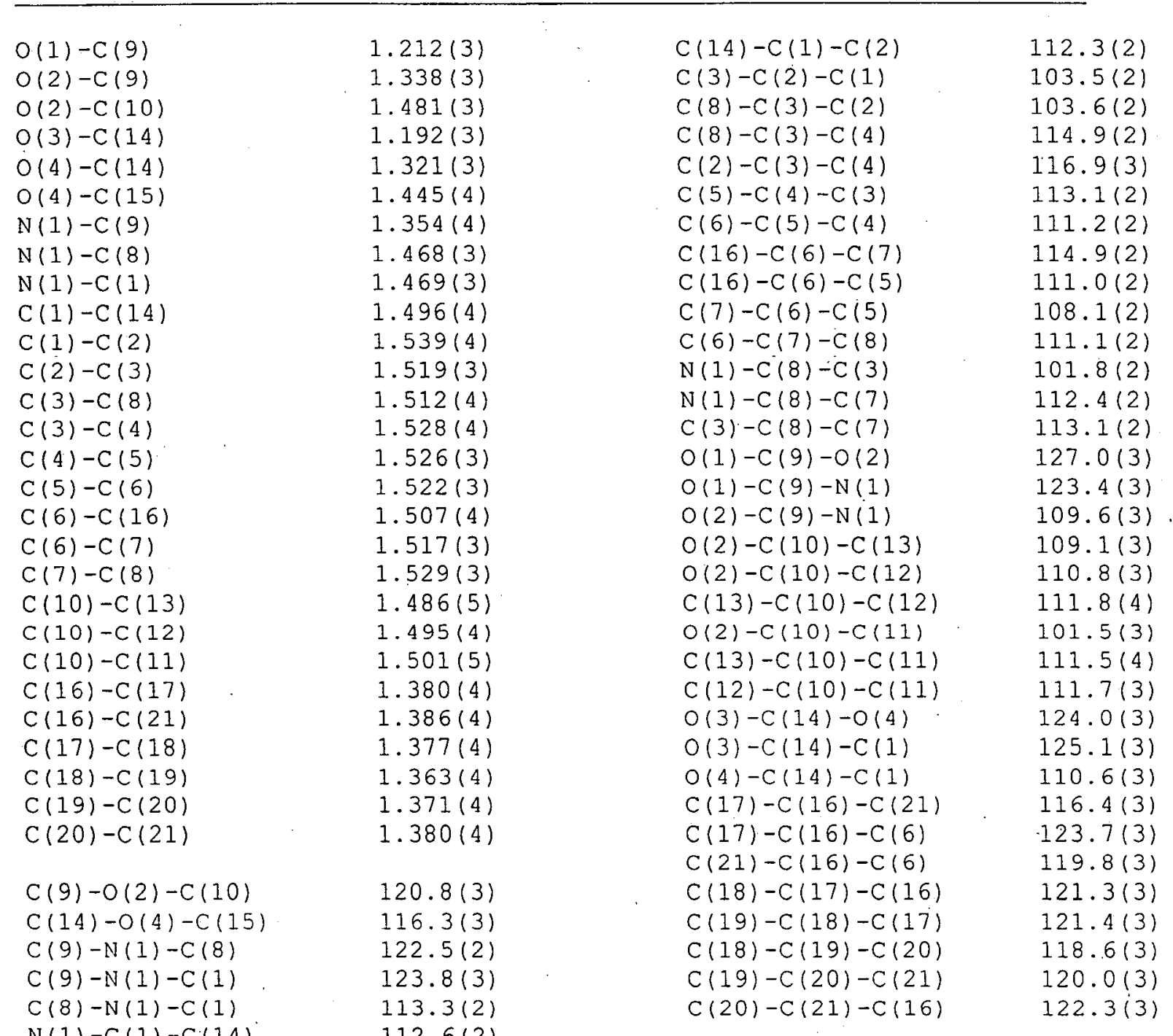

$\mathrm{N}(1)-\mathrm{C}(1)-\mathrm{C}(14) \quad 112.6(2)$

$\mathrm{N}(1)-\mathrm{C}(1)-\mathrm{C}(2)$ 
Table 6. Torsion angles [ ${ }^{\circ}$ ] for $\mathrm{C} 21$ H29 $\mathrm{N} 04$.

\begin{tabular}{|c|c|c|c|}
\hline$C(9)-N(1)-C(1)-C(14)$ & $-60.5(4)$ & $C(8)-N(1)-C(9)-O(1)$ & $-5.3(5)$ \\
\hline$C(8)-N(1)-C(1)-C(14)$ & $126.9(3)$ & $\mathrm{C}(1)-\mathrm{N}(1)-\mathrm{C}(9)-\mathrm{O}(1)$ & $-177.2(3)$ \\
\hline$C(9)-N(1)-C(1)-C(2)$ & $178.8(3)$ & $C(8)-N(1)-C(9)-O(2)$ & $174.0(2)$ \\
\hline$C(8)-N(1)-C(1)-C(2)$ & $6.2(3)$ & $C(1)-N(1)-C(9)-O(2)$ & $2.1(4)$ \\
\hline$N(1)-C(1)-C(2)-C(3)$ & $-27.9(3)$ & $C(9)-O(2)-C(10)-C(13)$ & $-60.1(4)$ \\
\hline$C(14)-C(1)-C(2)-C(3)$ & $-148.8(2)$ & $C(9)-O(2)-C(10)-C(12)$ & $63.4(4)$ \\
\hline$C(1)-C(2)-C(3)-C(8)$ & $39.8(3)$ & $C(9)-O(2)-C(10)-C(11)$ & $-177.9(3)$ \\
\hline$C(1)-C(2)-C(3)-C(4)$ & $167.3(2)$ & $C(15)-O(4)-C(14)-O(3)$ & $3.6(5)$ \\
\hline$C(8)-C(3)-C(4)-C(5)$ & $42.4(4)$ & $C(15)-O(4)-C(14)-C(1)$ & $177.9(3)$ \\
\hline$C(2)-C(3)-C(4)-C(5)$ & $-79.4(3)$ & $\mathrm{N}(1)-\mathrm{C}(1)-\mathrm{C}(14)-\mathrm{O}(3)$ & $-37.5(4)$ \\
\hline$C(3)-C(4)-C(5)-C(6)$ & $-52.0(3)$ & $C(2)-C(1)-C(14)-O(3)$ & $77.1(4)$ \\
\hline$C(4)-C(5)-C(6)-C(16)$ & $-171.5(2)$ & $\mathrm{N}(1)-\mathrm{C}(1)-\mathrm{C}(14)-\mathrm{O}(4)$ & $148.3(2)$ \\
\hline$C(4)-C(5)-C(6)-C(7)$ & $61.7(3)$ & $C(2)-C(1)-C(14)-O(4)$ & $-97.0(3)$ \\
\hline$C(16)-C(6)-C(7)-C(8)$ & $173.4(2)$ & $C(7)-C(6)-C(16)-C(17)$ & $36.3(4)$ \\
\hline$C(5)-C(6)-C(7)-C(8)$ & $-62.1(3)$ & $C(5)-C(6)-C(16)-C(17)$ & $-86.6(3)$ \\
\hline$C(9)-N(1)-C(8)-C(3)$ & $-154.7(3)$ & $C(7)-C(6)-C(16)-C(21)$ & $-147.4(3)$ \\
\hline$C(1)-N(1)-C(8)-C(3)$ & $18.0(3)$ & $C(5)-C(6)-C(16)-C(21)$ & $89.7(3)$ \\
\hline$C(9)-N(1)-C(8)-C(7)$ & $84.0(3)$ & $C(21)-C(16)-C(17)-C(18)$ & $-0.3(4)$ \\
\hline $\mathrm{C}(1)-\mathrm{N}(1)-\mathrm{C}(8)-\mathrm{C}(7)$ & $-103.3(3)$ & $C(6)-C(16)-C(17)-C(18)$ & $176.1(3)$ \\
\hline$C(2)-C(3)-C(8)-N(1)$ & $-34.9(3)$ & $C(16)-C(17)-C(18)-C(19)$ & $-0.3(5)$ \\
\hline$C(4)-C(3)-C(8)-N(1)$ & $-163.6(2)$ & $C(17)-C(18)-C(19)-C(20)$ & $0.6(5)$ \\
\hline$C(2)-C(3)-C(8)-C(7)$ & $85.9(3)$ & $C(18)-C(19)-C(20)-C(21)$ & $-0.2(5)$ \\
\hline$C(4)-C(3)-C(8)-C(7)$ & $-42.8(3)$ & $C(19)-C(20)-C(21)-C(16)$ & $-0.5(5)$ \\
\hline$C(6)-C(7)-C(8)-N(1)$ & $167.8(2)$ & $C(17)-C(16)-C(21)-C(20)$ & $0.7(4)$ \\
\hline$C(6)-C(7)-C(8)-C(3)$ & $53.2(3)$ & $C(6)-C(16)-C(21)-C(20)$ & $-175.8(3)$ \\
\hline$C(10)-O(2)-C(9)-O(1)$ & $-4.0(5)$ & & \\
\hline$C(10)-O(2)-C(9)-N(1)$ & $176.8(3)$ & & \\
\hline
\end{tabular}




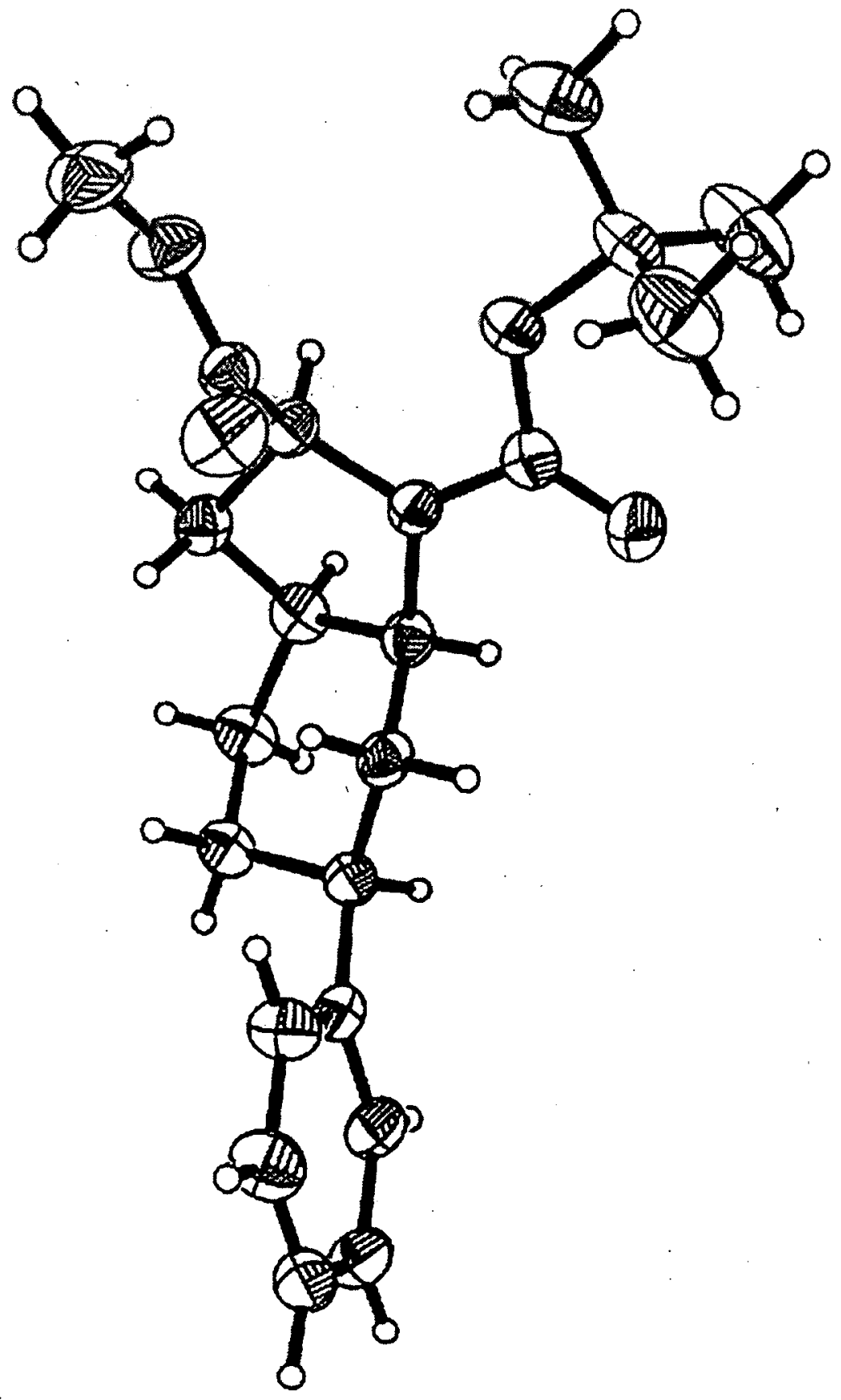

ORTEP view of the C21 H29 N O4 compound with the numbering scheme adopted. Ellipsoids drawn at $30 \%$ probability level. Hydrogens represented by sphere of arbitrary size. 
뭌 (C) 2004 American Chemical Society, Org. Lett., Hanessian ol040053b Supporting Info Page 28

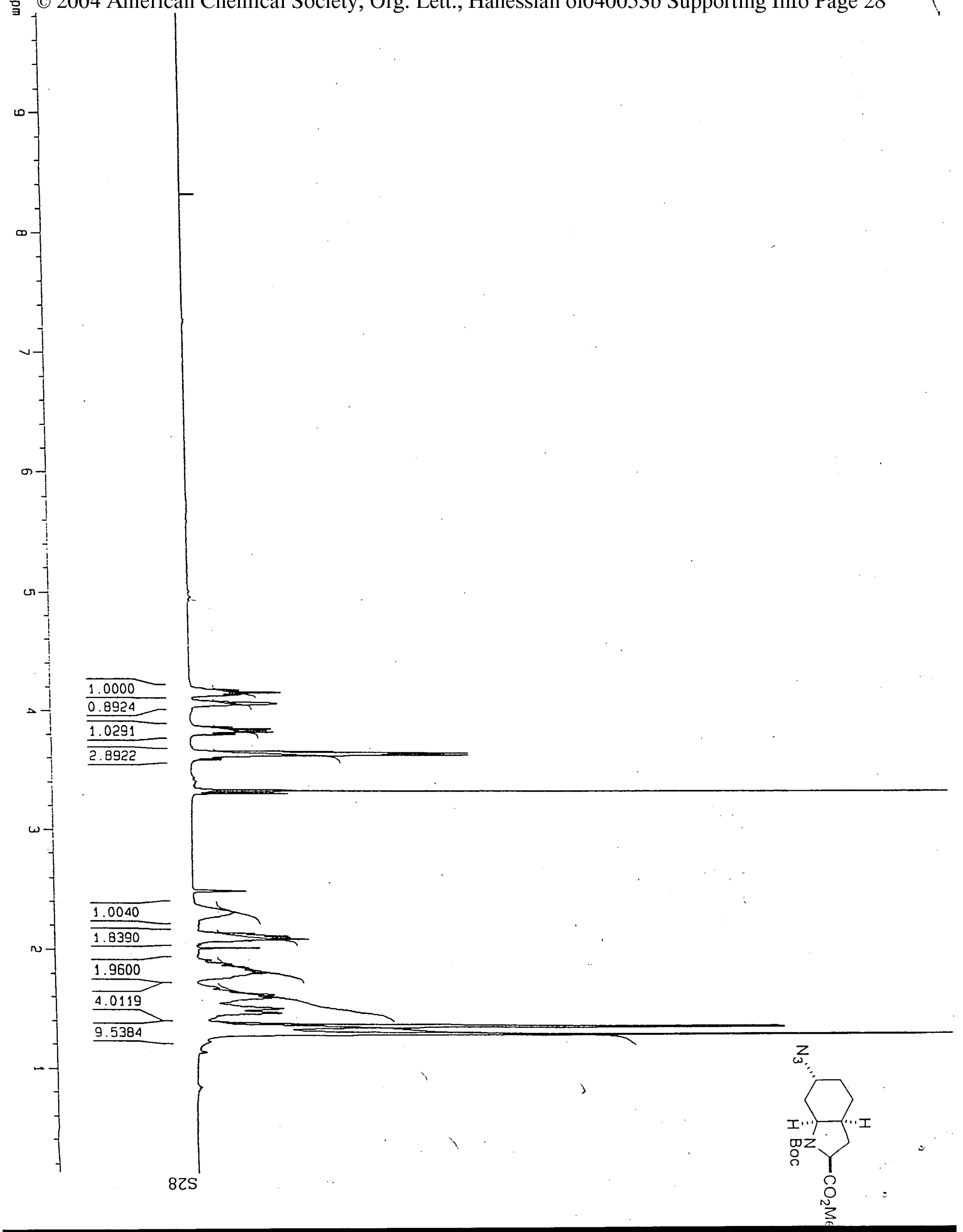


의 3

(C) 2004 American Chemical Society, Org. Lett., Hanessian ol040053b Supporting Info Page 29

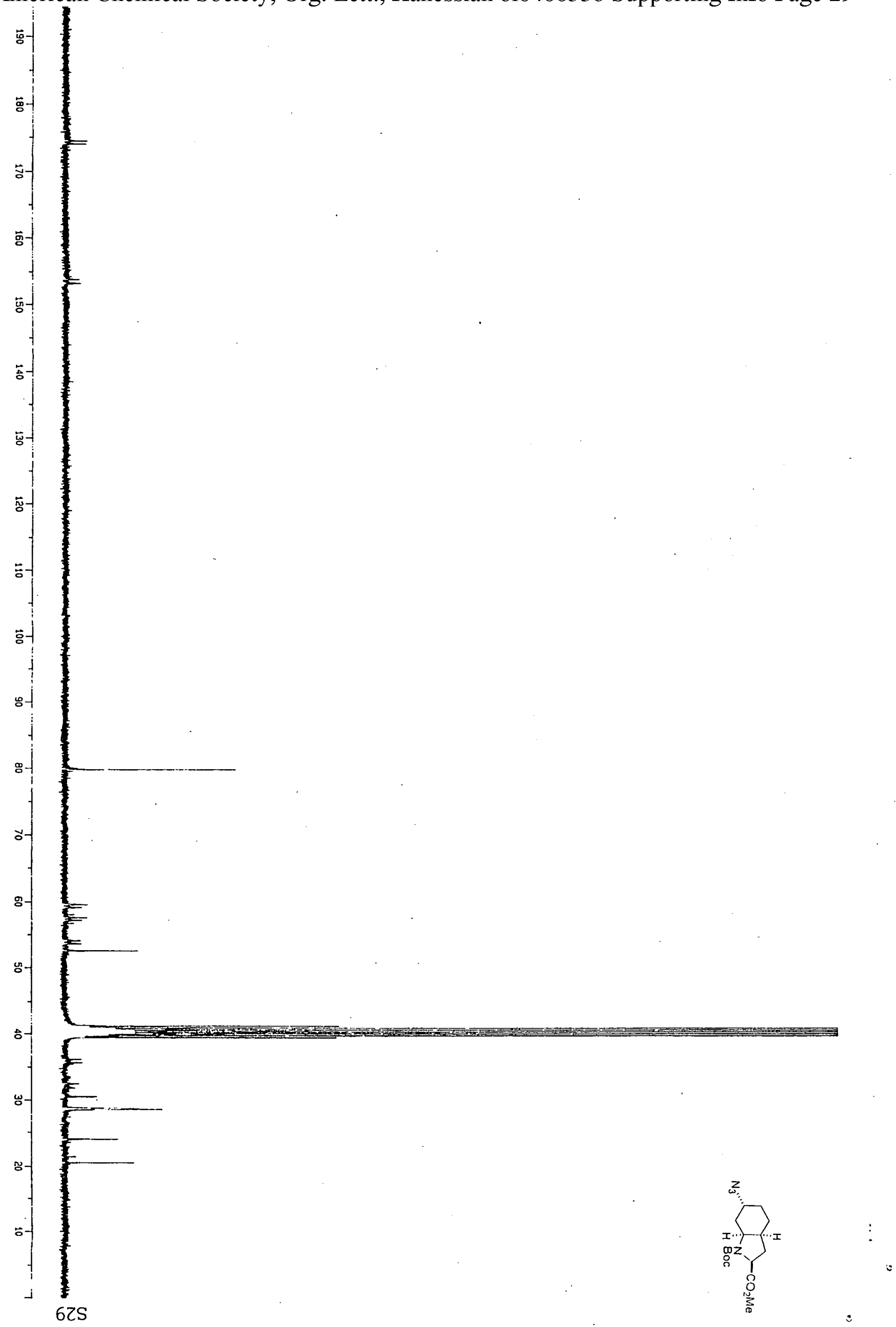


(C) 2004 American Chemical Society, Org. Lett., Hanessian ol040053b Supporting Info Page 30
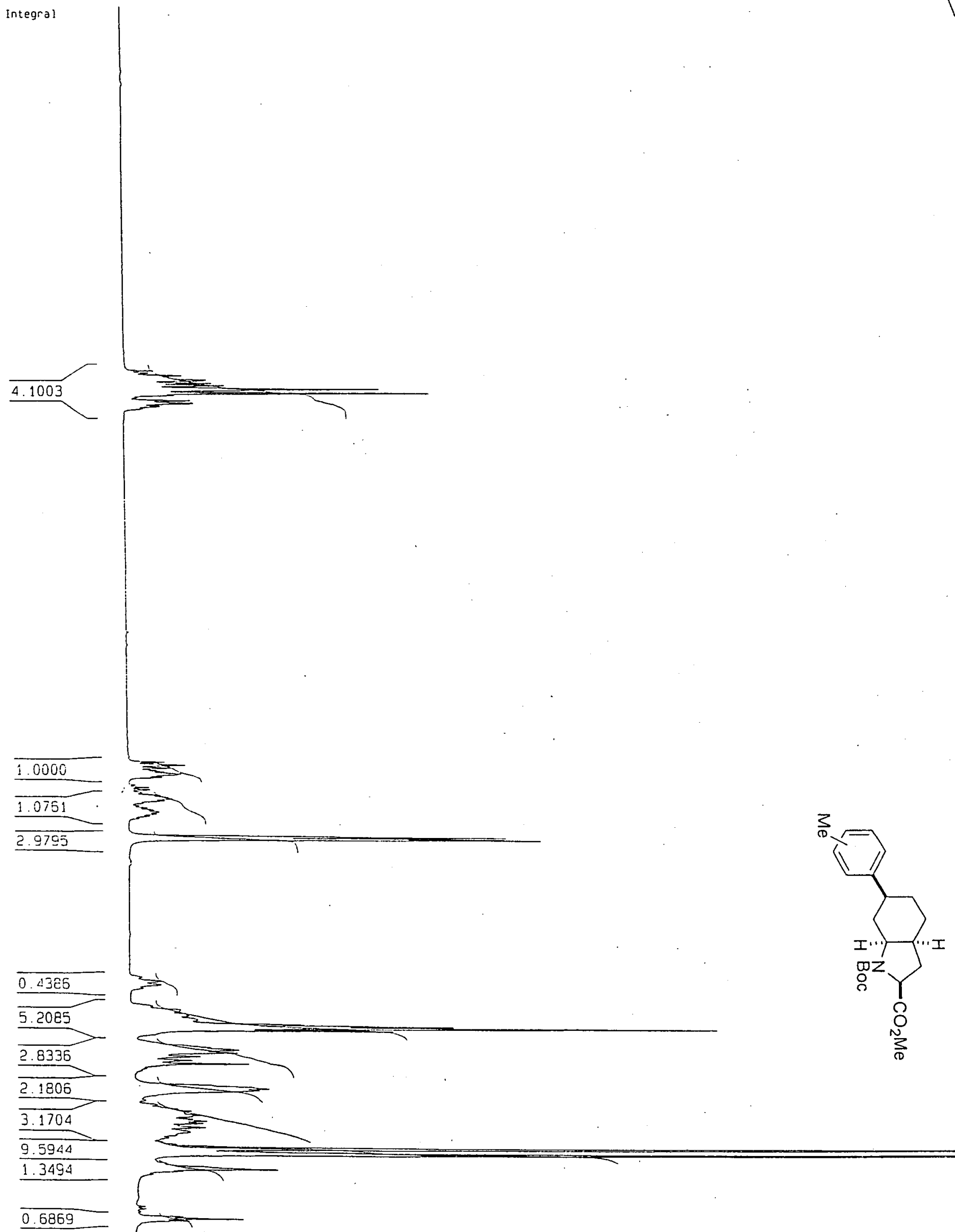

OES 
(C) 2004 American Chemical Society, Org. Lett., Hanessian ol040053b Supporting Info Page 31 를

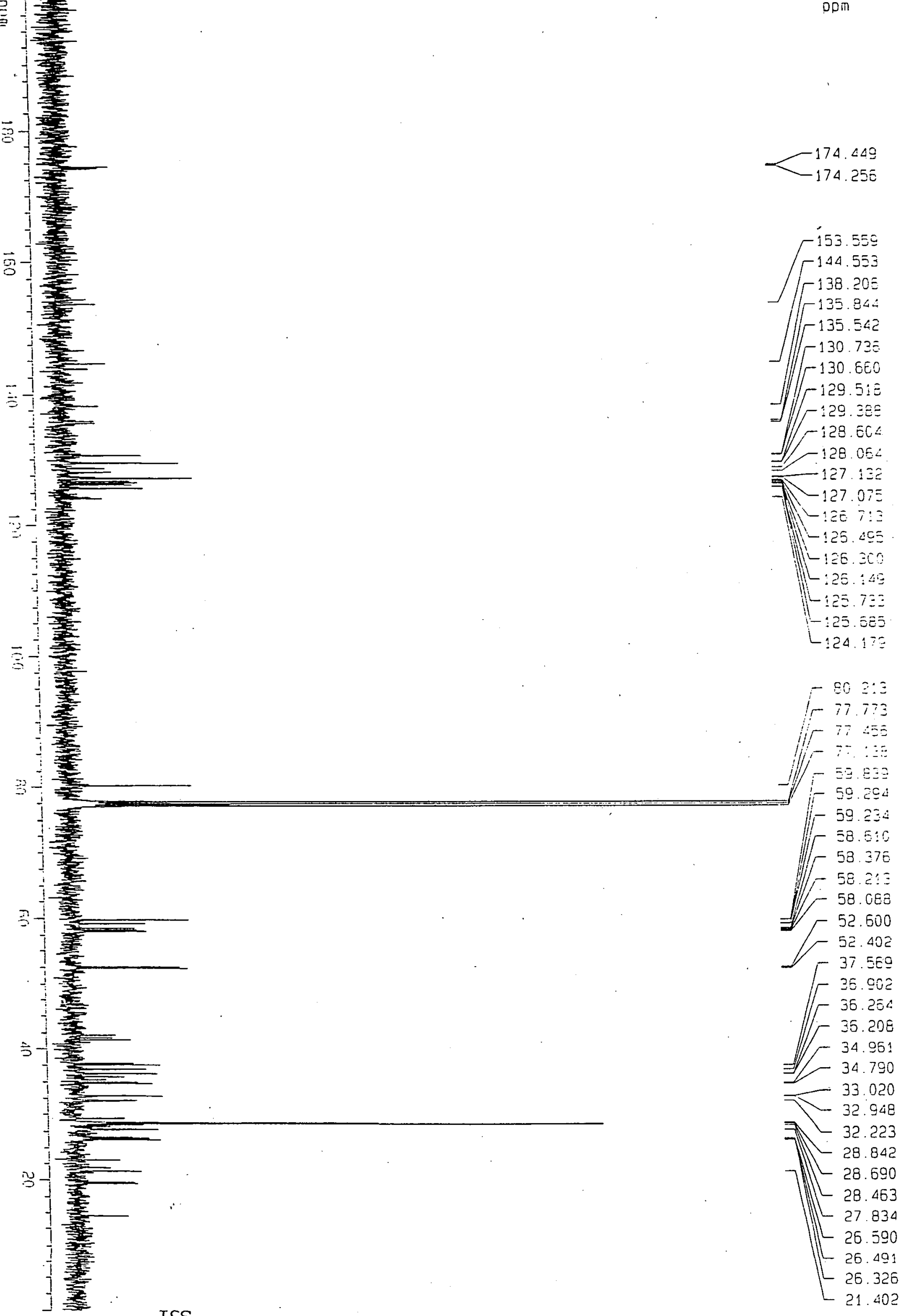


(C) 2004 American Chemical Society, Org. Lett., Hanessian ol040053b Supporting Info Page 32

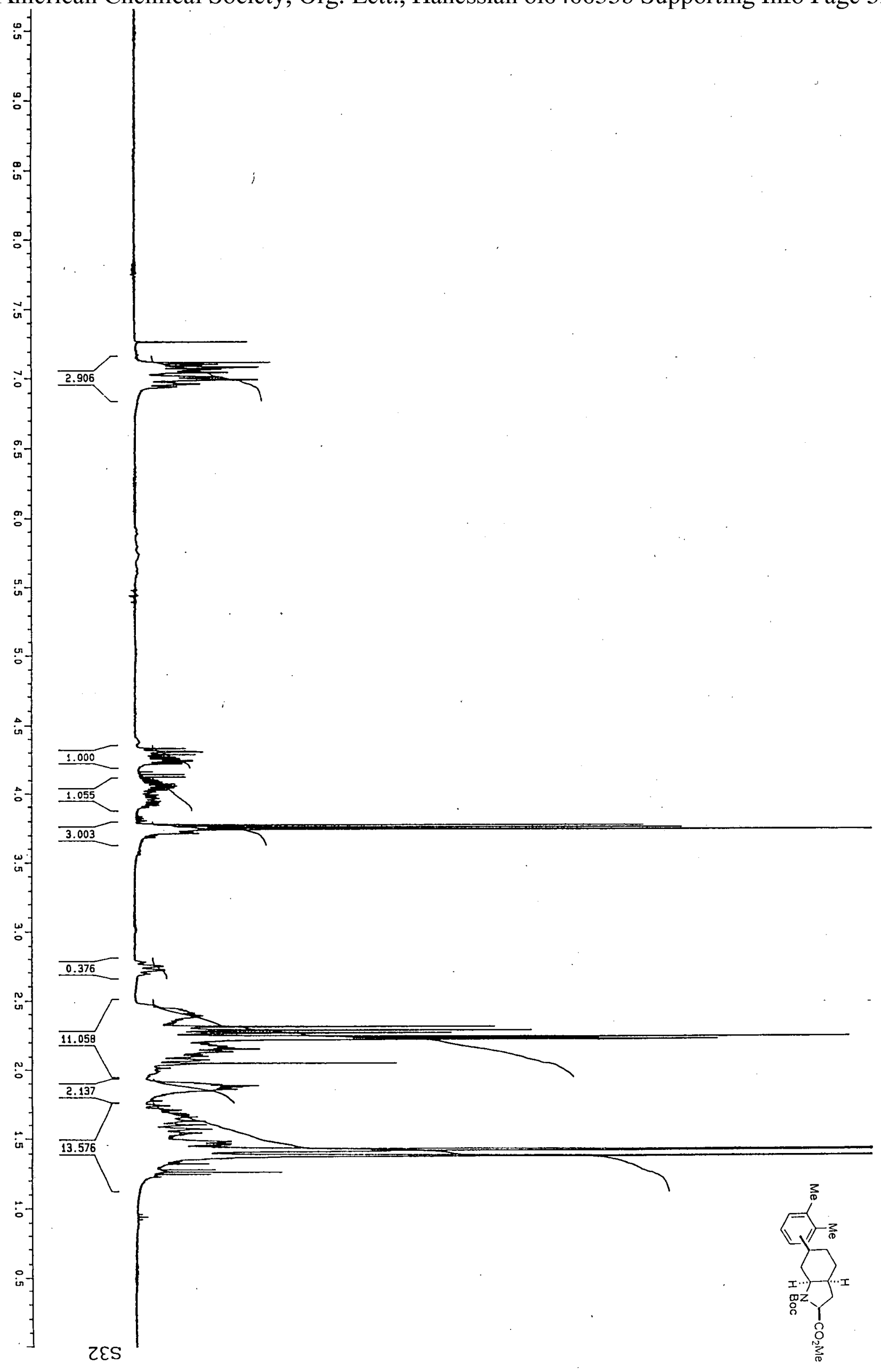




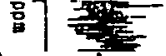

(c) 2004 American Chemical Society, Org. Lett., Hanessian ol040053b Supporting Info Page 33

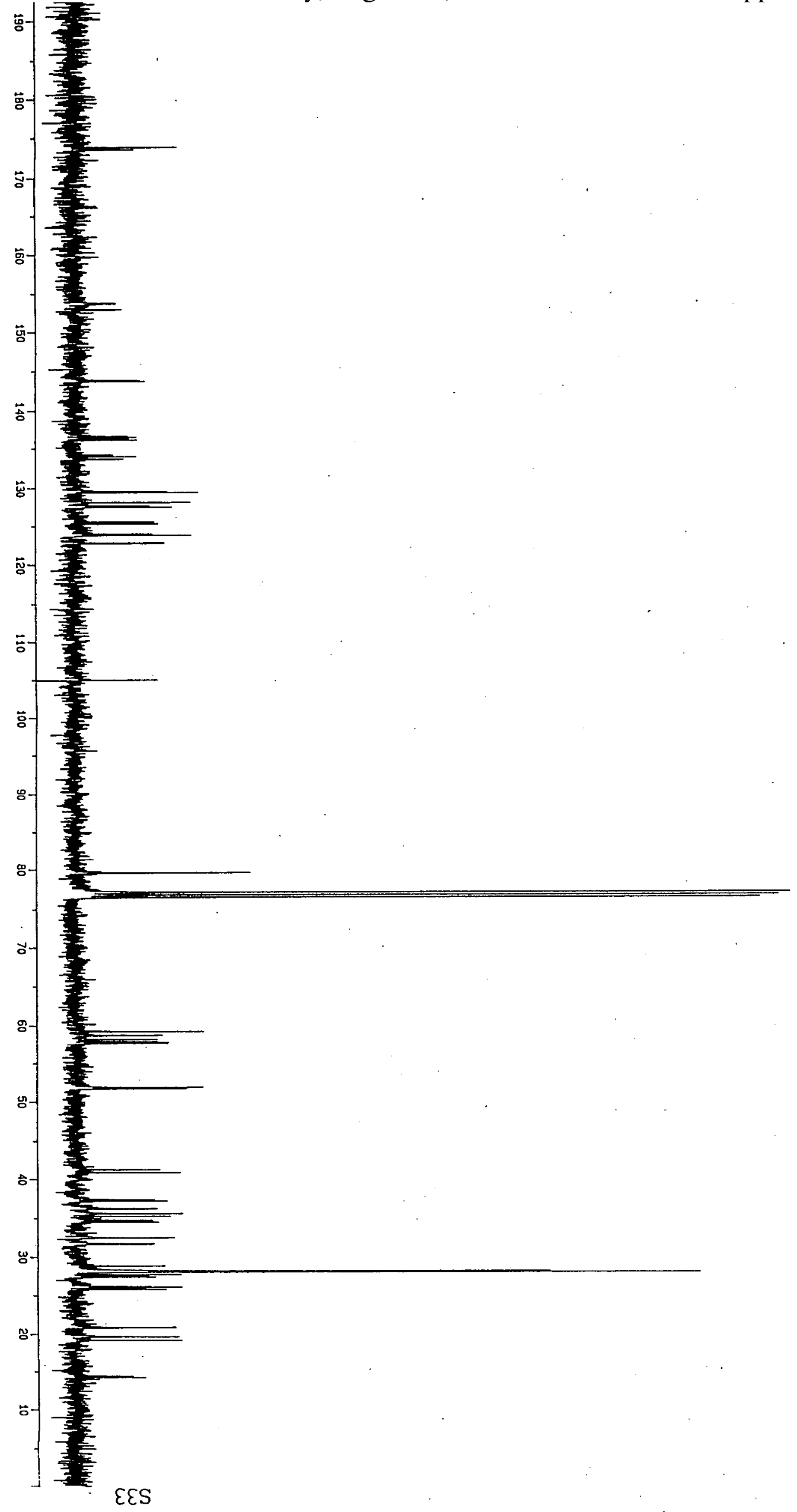




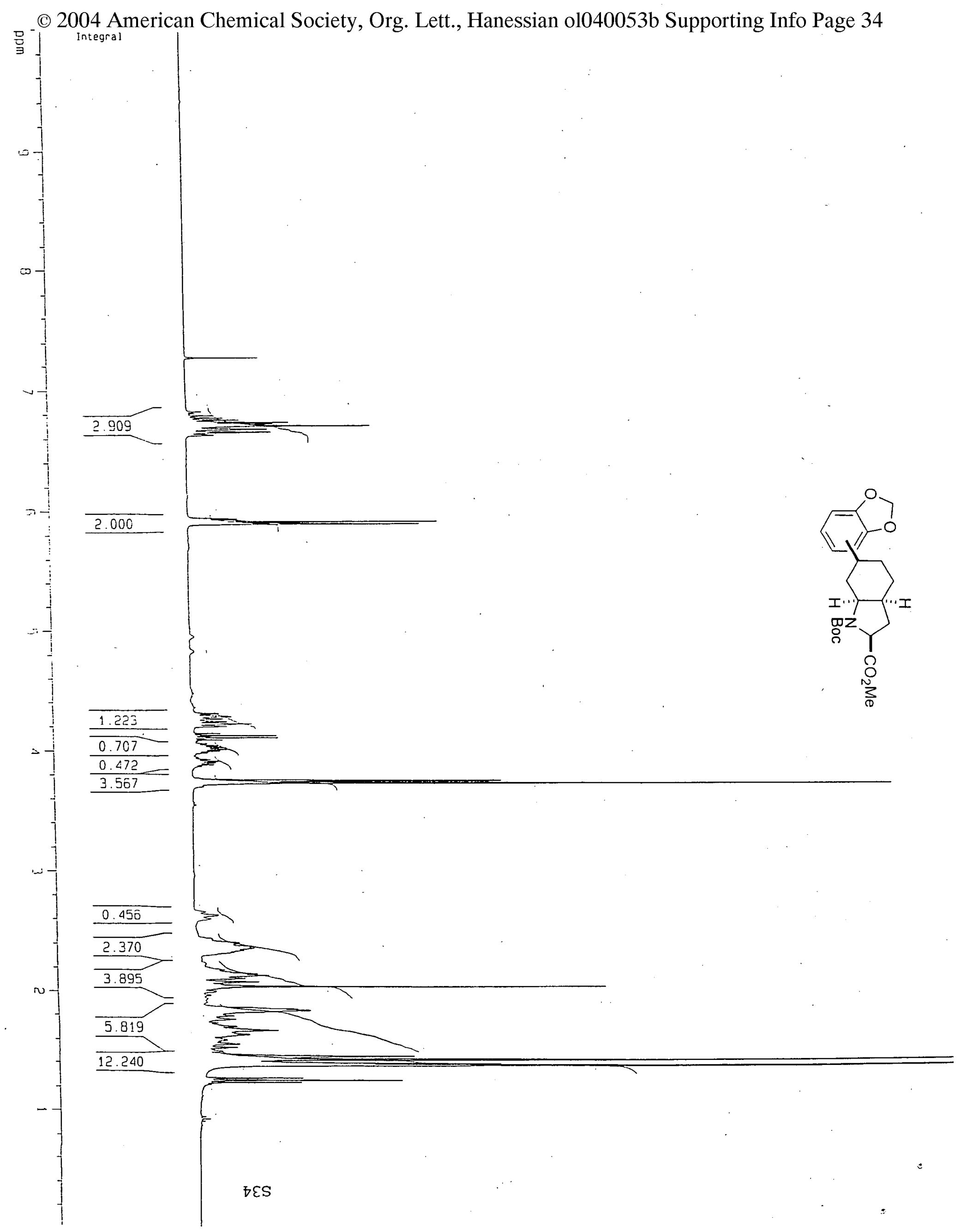


(C) 2004 American Chemical Society, Org. Lett., Hanessian ol040053b Supporting Info Page 35

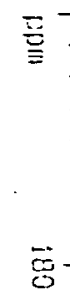

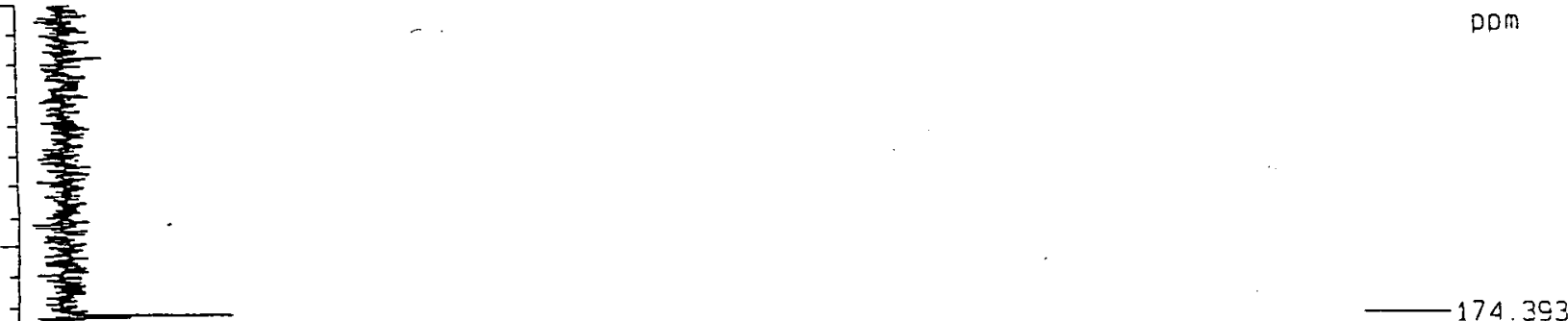

174.393

$\Gamma^{154.331}$

$\tau^{153.534}$

$\Rightarrow \Gamma^{147.881}$

$\int \Gamma^{140.782}$

$-128.172$

-122.008

$-121.854$

$-120.194$

-119.94?

$\sqrt{ } / 119.822$

-108.557

III $\Gamma^{108.453}$

= $-107.80 !$

$-107.754$

-106. 939

IIf -101.214

$\exists /{ }^{101.132}$

$-100.87:$

$=\frac{1}{1}$

然

$-100.825$

$-80.233$

F 80.203

$1+77.770$

$-77.452$

$\sqrt{1 / 57.125}$

9

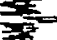

$1 / 50.792$

T5.829

.

를

59.796

T59.265

T 58.018

$-57.796$

- 52.574

$\exists / 52.389$

છ

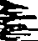

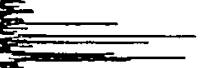

$-.52 .355$

r 41.957

₹

$\Gamma 41.713$

r 36.804

$\int \Gamma^{36.396}$

今-

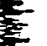

룰

$-36.220$

36.141

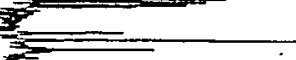

- 35.878

$-35.716$

$-32.921$

$-32.084$

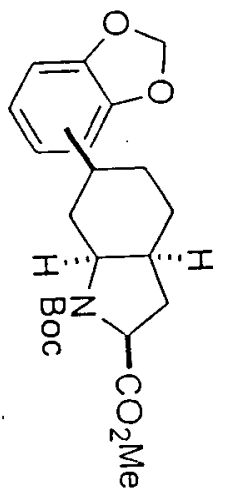

r

롤

$-28.849$

$L 28.678$

$-26.644$

$-26.385$

$[26.247$

L 26.162 
(C) 2004 American Chemical Society, Org. Lett., Hanessian ol040053b Supporting Info Page 36
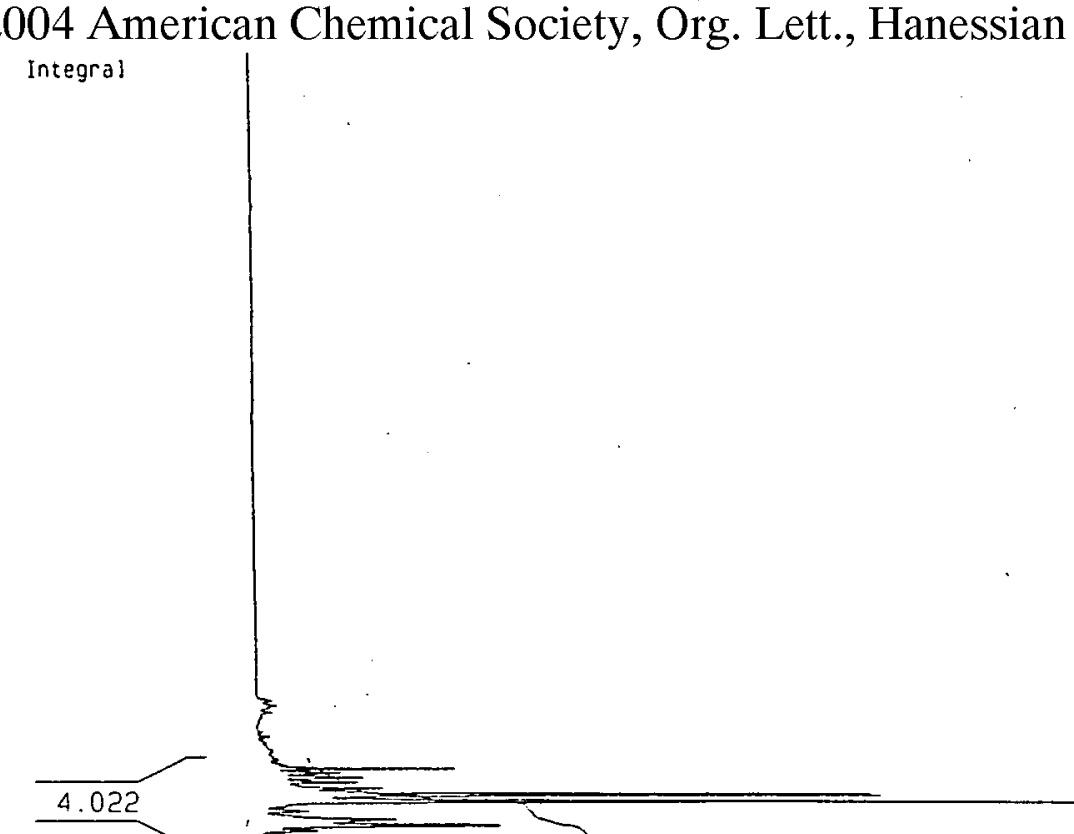

$\checkmark$

4.022

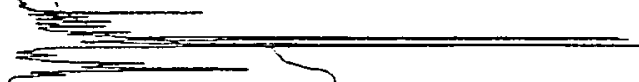

$=$

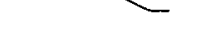

1.000
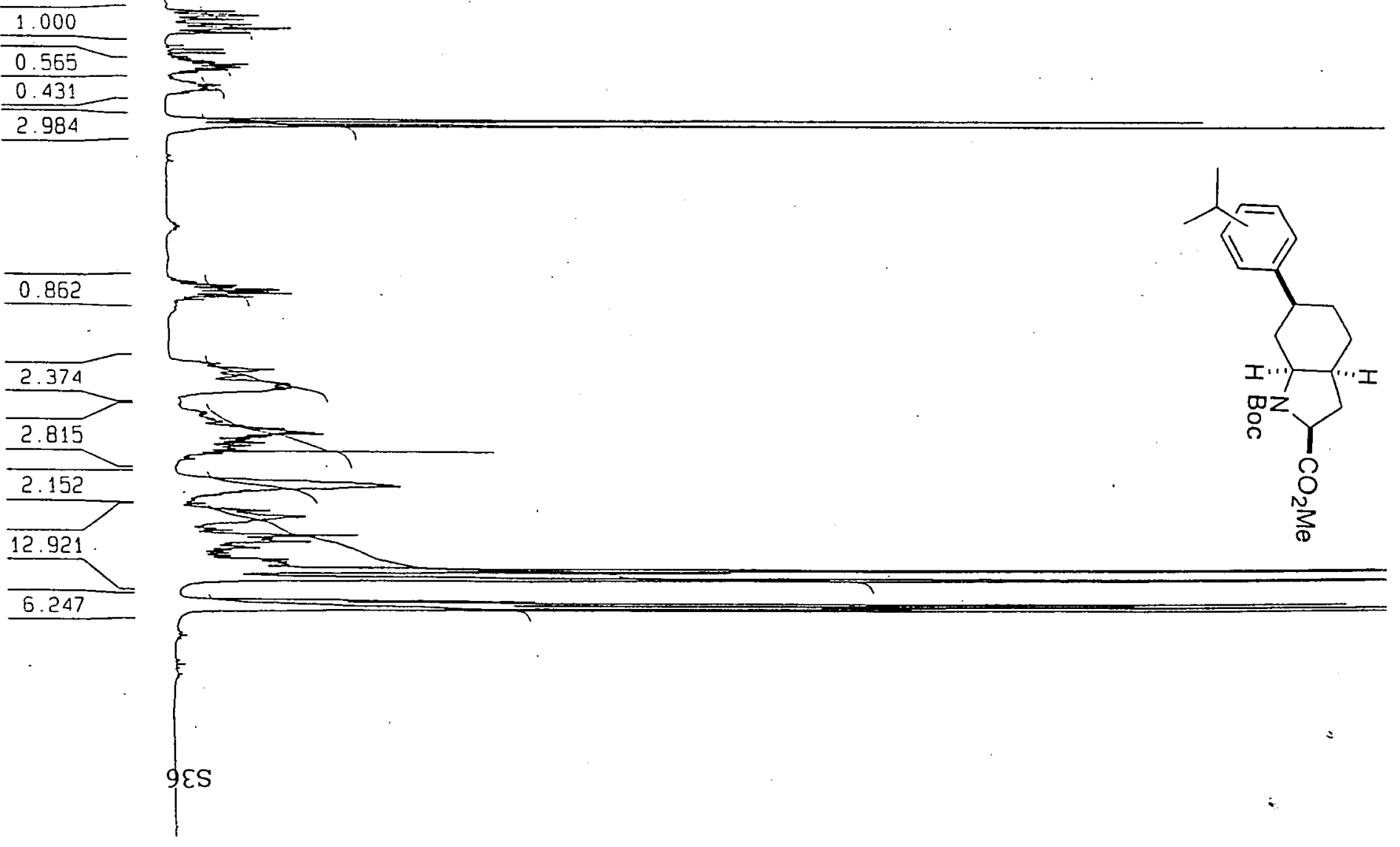
(C) 2004 American Chemical Society, Org. Lett., Hanessian ol040053b Supporting Info Page 37

믈

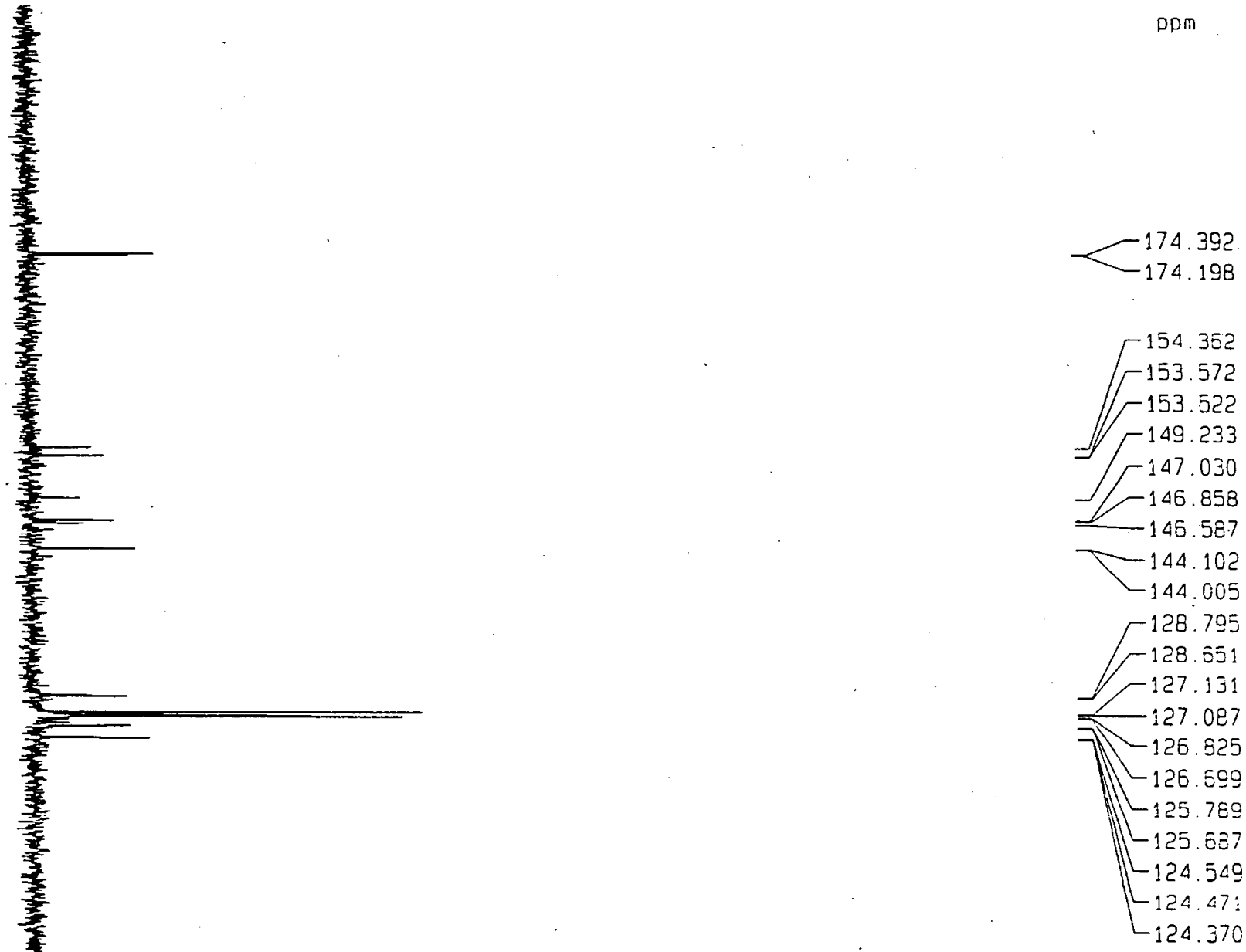

亏-

然

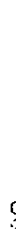

I
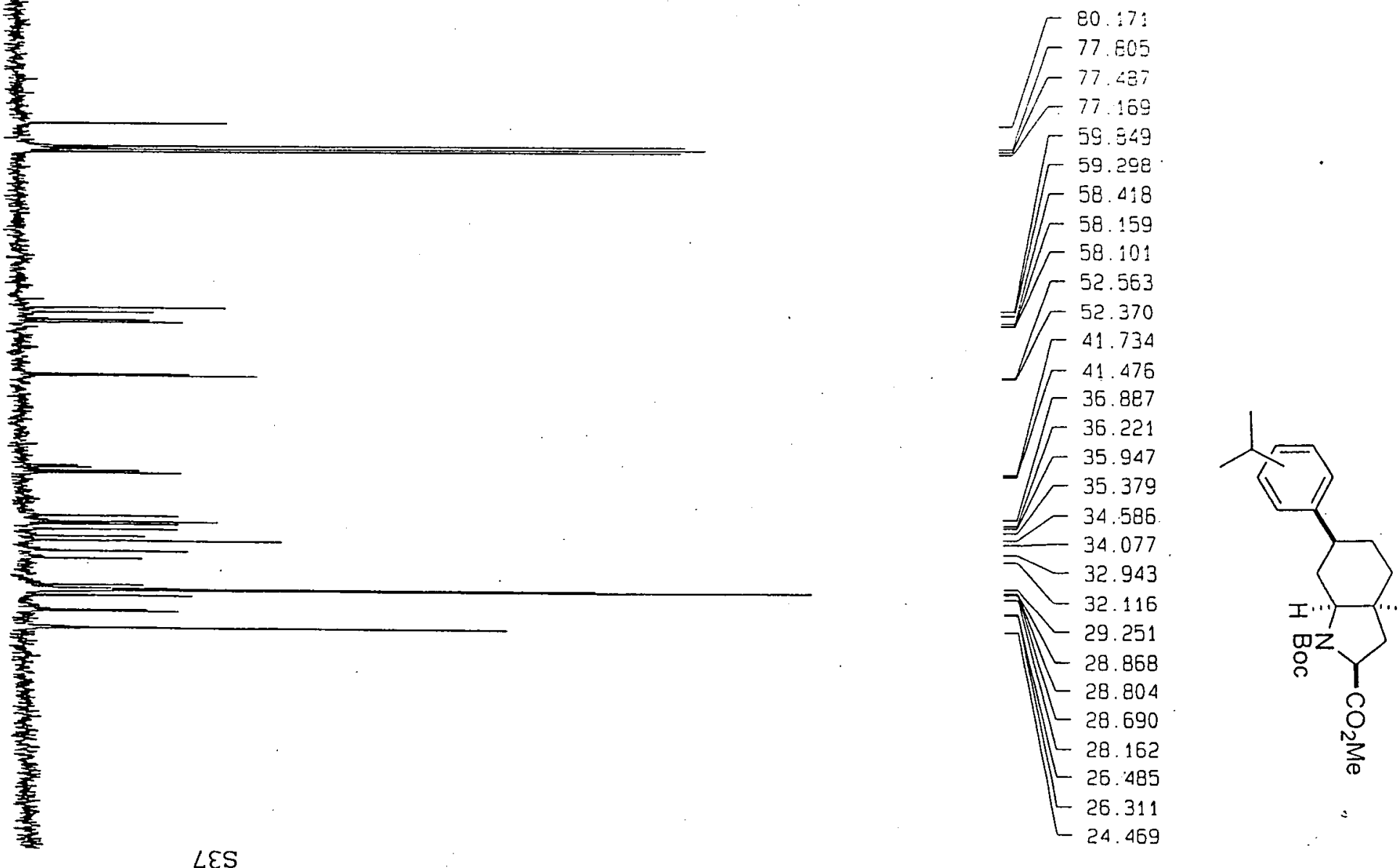
(c) 2004 American Chemical Society, Org. Lett., Hanessian ol040053b Supporting Info Page 38

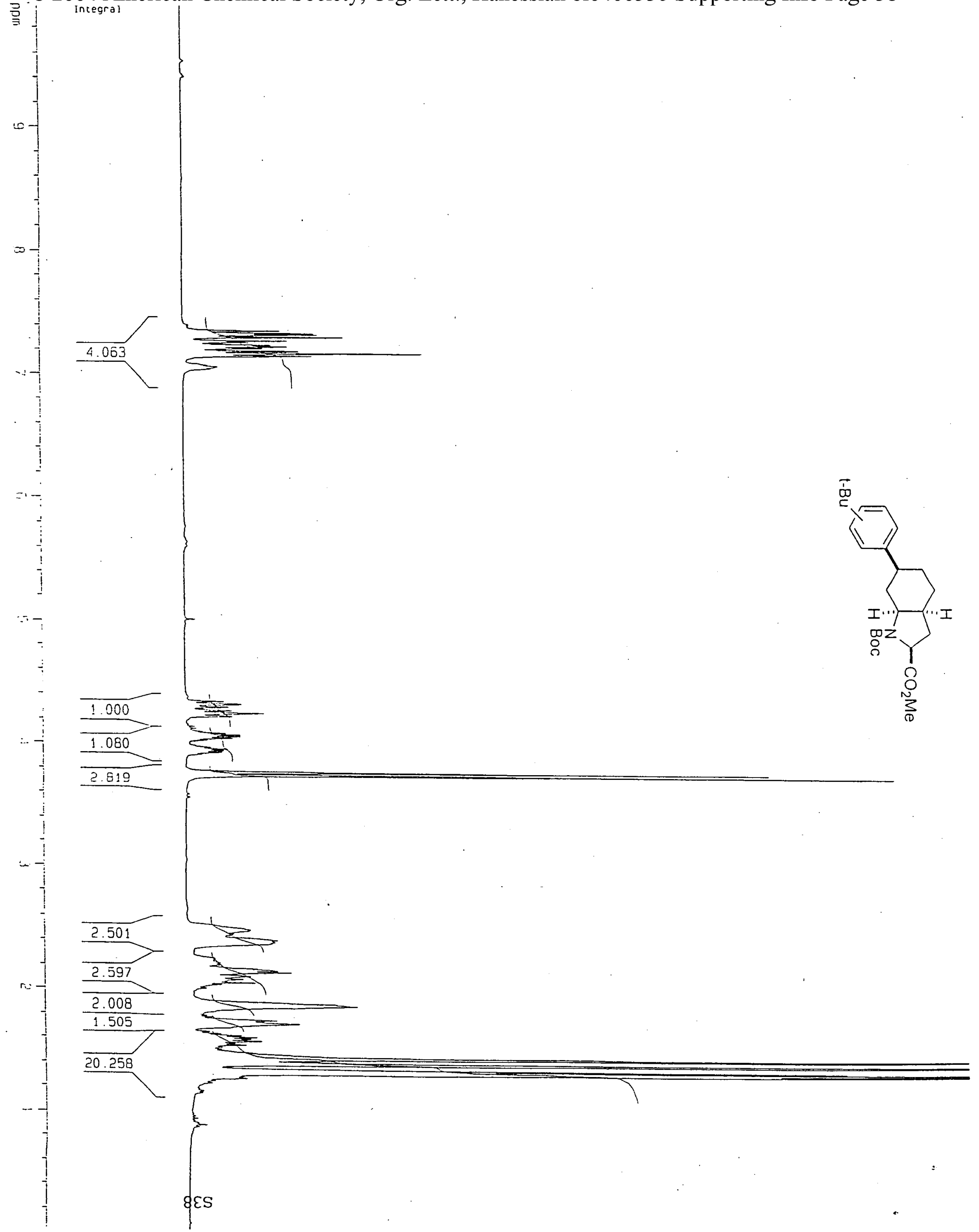


(C) 2004 American Chemical Society, Org. Lett., Hanessian ol040053b Supporting Info Page 39 罗

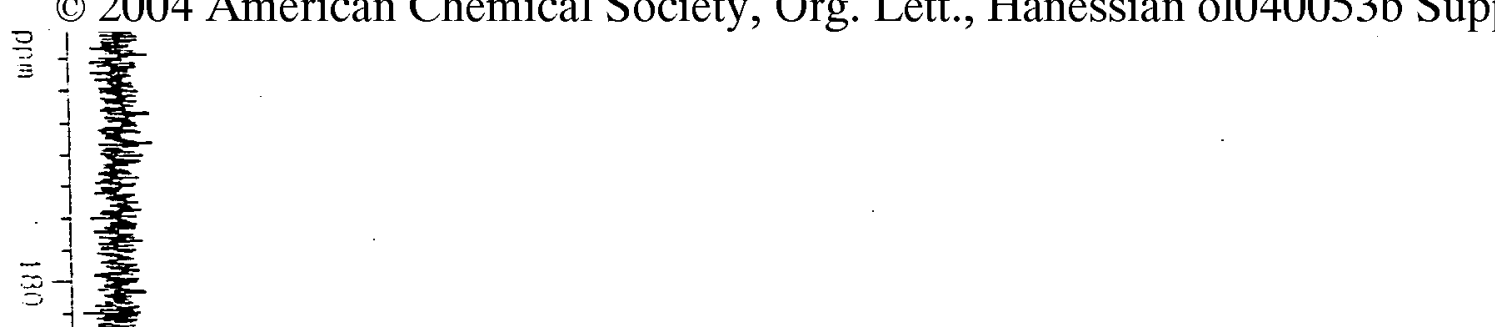


(C) 2004 American Chemical Society, Org. Lett., Hanessian ol040053b Supporting Info Page 40

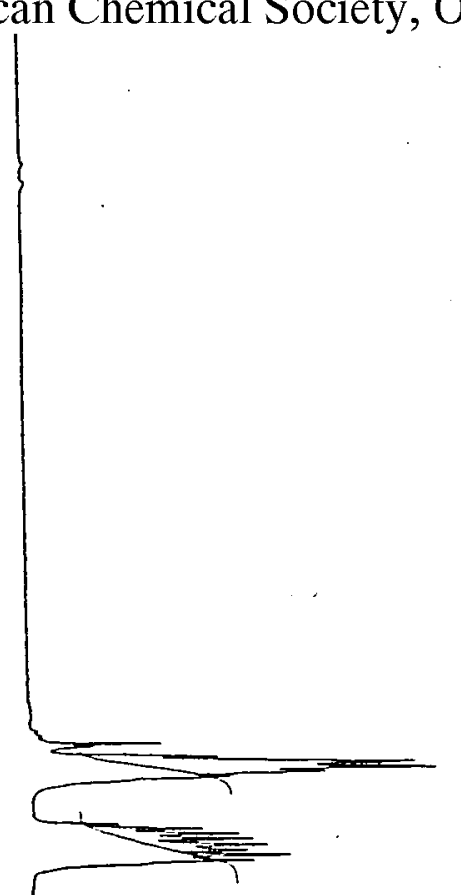

$\checkmark$
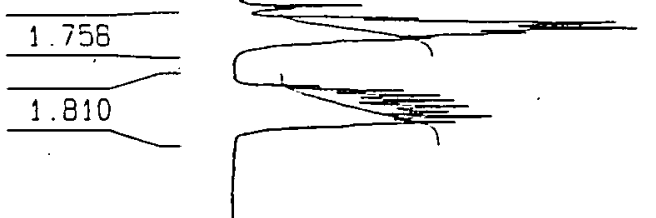

$=$
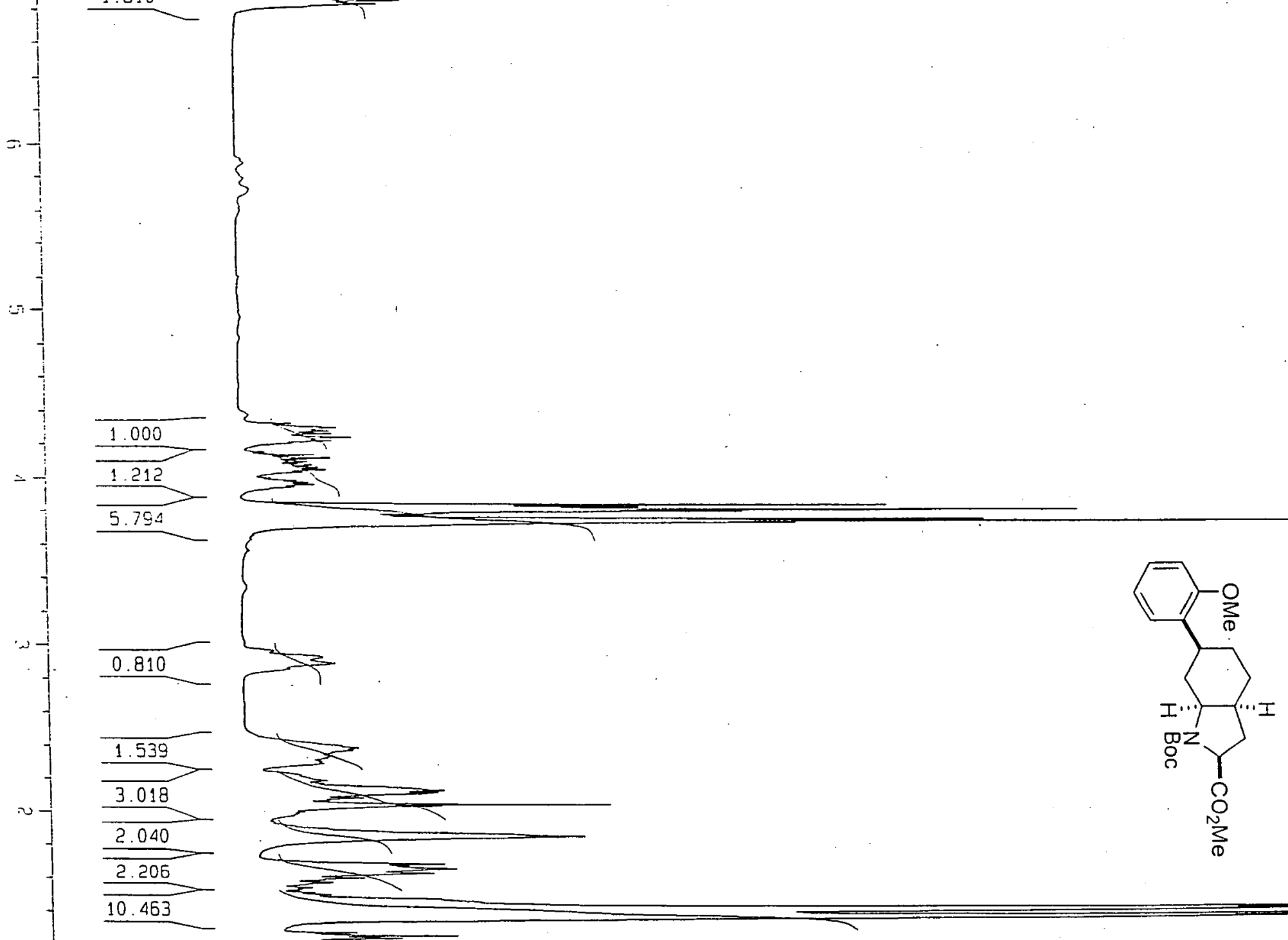